\title{
Generalized Spatial Modulation in Large-Scale Multiuser MIMO Systems
}

\author{
T. Lakshmi Narasimhan, P. Raviteja, and A. Chockalingam \\ Department of ECE, Indian Institute of Science, Bangalore-560012, India
}

\begin{abstract}
Generalized spatial modulation (GSM) uses $n_{t}$ transmit antenna elements but fewer transmit radio frequency (RF) chains, $n_{r f}$. Spatial modulation (SM) and spatial multiplexing are special cases of GSM with $n_{r f}=1$ and $n_{r f}=n_{t}$, respectively. In GSM, in addition to conveying information bits through $n_{r f}$ conventional modulation symbols (for example, QAM), the indices of the $n_{r f}$ active transmit antennas also convey information bits. In this paper, we investigate GSM for large-scale multiuser MIMO communications on the uplink. Our contributions in this paper include: $(i)$ an average bit error probability (ABEP) analysis for maximum-likelihood detection in multiuser GSM-MIMO on the uplink, where we derive an upper bound on the ABEP, and (ii) low-complexity algorithms for GSMMIMO signal detection and channel estimation at the base station receiver based on message passing. The analytical upper bounds on the ABEP are found to be tight at moderate to high signal-tonoise ratios (SNR). The proposed receiver algorithms are found to scale very well in complexity while achieving near-optimal performance in large dimensions. Simulation results show that, for the same spectral efficiency, multiuser GSM-MIMO can outperform multiuser SM-MIMO as well as conventional multiuser MIMO, by about 2 to $9 \mathrm{~dB}$ at a bit error rate of $10^{-3}$. Such SNR gains in GSM-MIMO compared to SM-MIMO and conventional MIMO can be attributed to the fact that, because of a larger number of spatial index bits, GSM-MIMO can use a lower-order QAM alphabet which is more power efficient.
\end{abstract}

Keywords - Large-scale MIMO systems, generalized spatial modulation, GSM-MIMO receiver, channel hardening, message passing.

\section{INTRODUCTION}

Large-scale MIMO systems with tens to hundreds of antennas are getting increased research attention [1]-[3]. Because of its advantages of very high spectral efficiencies/sum rates, increased reliability, and power efficiency, large-scale MIMO technology is being considered as a potential technology for next generation (example, 5G) wireless systems [4]. The following two characteristics are typical in conventional MIMO systems: $(i)$ there will be one transmit radio frequency (RF) chain for each transmit antenna (i.e., if $n_{t}$ is the number of transmit antennas, then the number of transmit RF chains, $n_{r f}$, will also be $n_{t}$ ), and (ii) information bits are carried only on the modulation symbols (example, QAM). Conventional multiuser MIMO systems with a large number (tens to hundreds) of antennas at the base station (BS) are referred to as 'massive MIMO' systems in the recent literature [3], [4]. Key technological issues that need to be addressed in practical realization of large-scale MIMO systems include design and placement of compact antennas, multiple RF chains, and largedimension transmit/receive signal processing techniques and algorithms [1]-[3].
Spatial modulation (SM), an attractive modulation scheme for multi-antenna communications [5],[6], can alleviate the requirement of multiple transmit RF chains in MIMO systems. In SM, the transmitter has multiple transmit antennas but only one transmit RF chain. In a given channel use, only one of the $n_{t}$ transmit antennas will be activated, and the remaining $n_{t}-1$ antennas remain silent. On the active transmit antenna, a symbol from a conventional modulation alphabet $\mathbb{A}$ is transmitted. In addition to information bits conveyed through the modulation symbol from $\mathbb{A}$, the index of the active transmit antenna also conveys information bits. Therefore, the number of bits conveyed in one channel use in SM is $\left\lfloor\log _{2} n_{t}\right\rfloor+\left\lfloor\log _{2}|\mathbb{A}|\right\rfloor$. Space shift keying (SSK) is a special case of SM. Like in SM, in SSK also only one antenna among $n_{t}$ antennas is activated in a given channel use. On the activated antenna, instead of sending a symbol from a conventional alphabet as is done in SM, a constant amplitude signal (say, +1) is transmitted in SSK. Therefore, the number of information bits conveyed in one channel use in SSK is $\left\lfloor\log _{2} n_{t}\right\rfloor$. For example, for $n_{t}=4$, the two-bit combinations $\{00,01,10,11\}$ are mapped to antenna indices $\{1,2,3,4\}$; antenna 1 is activated when input bits are 00 , antenna 2 is activated when input bits are 01 , antenna 3 is activated when input bits are 10, and antenna 4 is activated when input bits are 11. The problem of SSK signal detection at the receiver in a given channel use then becomes one of finding which one among the $n_{t}$ antennas was activated, i.e., determining the index of the active antenna. Assuming that the choice of an active antenna among all antennas is equally likely and a ' +1 ' was sent on the active antenna, and that the channel gains from $n_{t}$ transmit antennas to $n_{r}$ receive antennas are known at the receiver, the maximum likelihood (ML) decision rule to find the active antenna index is given by

$$
\hat{j}=\arg \min _{j, 1 \leq j \leq n_{t}}\left\|\mathbf{y}-\mathbf{h}_{j}\right\|^{2},
$$

where $\mathbf{y}$ is the $n_{r} \times 1$ received signal vector and $\mathbf{h}_{j}$ is the $n_{r} \times 1$ channel gain vector from transmit antenna $j$ to the receive antennas. The estimated antenna index $\hat{j}$ is then demapped to the information bits which represent that index. In SM signal detection, in addition to detecting the active antenna index, information bits conveyed through the conventional modulation symbol carried on the active antenna also have to be detected.

A lot of recent research has focused on SM and SSK in point-to-point as well as cooperative relaying settings (see [6], [7] and the references therein). Bit error performance of 
SSK and SM in single-user point-to-point communication has been analyzed in [8],[9]. Transmit diversity schemes for SM MIMO (i.e., systems that combine SM and space-time coding) have been analyzed in [10]. Multiuser SM MIMO on the downlink has been analyzed in [11]. SSK and SM employed on the uplink in multiuser MIMO systems have been studied in [12]-[16]. In [13]-[16], it has been shown that, for the same spectral efficiency, multiuser SM-MIMO can outperform conventional multiuser MIMO. In this paper, we are interested in a modulation scheme which is a generalization of SM, referred to as generalized spatial modulation (GSM) [17],[18]. GSM was introduced in [17] and its achievable rate was studied in detail in [18]. Here, we are interested in the performance analysis and signal detection of multiuser GSM on the uplink in large-scale MIMO systems. Such a study has not been reported in the literature before.

In GSM, the number of transmit RF chains, $n_{r f}$, is parameterized such that $1 \leq n_{r f} \leq n_{t}$, and, in a given channel use, $n_{r f}$ out of $n_{t}$ transmit antennas are chosen and activated [17],[18],[1]. The remaining $n_{t}-n_{r f}$ antennas remain silent. On the chosen antennas, $n_{r f}$ modulation symbols (one on each chosen antenna) are transmitted. The indices of the $n_{r f}$ active antennas out of $n_{t}$ available antennas convey $\left\lfloor\log _{2}\left(\begin{array}{c}n_{t} \\ n_{r f}\end{array}\right)\right\rfloor$ information bits. This is in addition to the $n_{r f}\left\lfloor\log _{2}|\mathbb{A}|\right\rfloor$ information bits conveyed by the $n_{r f}$ modulation symbols. It can be seen that both SM and spatial multiplexing turn out to be special cases of GSM with $n_{r f}=1$ and $n_{r f}=n_{t}$, respectively. In [18], it has been shown that for a given modulation alphabet and $n_{t}$, there exists an optimum $n_{r f}$ in GSM that maximizes the spectral efficiency.

In this paper, we consider the uplink in multiuser MIMO systems, where the BS has a large number of receive antennas (tens to hundreds) and each user terminal employs GSM with $n_{t}$ transmit antennas and $n_{r f}$ transmit RF chains. Some GSM configurations of interest at the user terminal include: $\left(n_{t}=4\right.$, $\left.n_{r f}=2\right),\left(n_{t}=8, n_{r f}=2\right)$. When $n_{t}>1$ and $n_{r f}=1$, GSM specializes to SM; example, $\left(n_{t}=2, n_{r f}=1\right),\left(n_{t}=4\right.$, $\left.n_{r f}=1\right)$. When $n_{t}=1$ and $n_{r f}=1$, GSM specializes to conventional modulation. Our contributions in this paper can be summarized as follows.

- We first analyze the average bit error probability (ABEP) of multiuser GSM-MIMO under maximum-likelihood (ML) detection. We derive an upper bound on the ABEP, which is tight at moderate to high signal-to-noise ratios (SNR).

- We then propose low-complexity algorithms for GSMMIMO signal detection and channel estimation at the BS receiver based on message passing. The proposed receiver algorithms scale very well in complexity and achieve near-ML performance in large dimensions. Simulation results show that, for the same spectral efficiency, multiuser GSM-MIMO can outperform multiuser SM-MIMO as well as conventional multiuser MIMO, by about 2 to 9 $\mathrm{dB}$ at a bit error rate (BER) of $10^{-3}$. Such SNR gains in GSM-MIMO compared to SM-MIMO and conventional MIMO can be attributed to the fact that, because of a larger number of spatial index bits, GSM-MIMO can

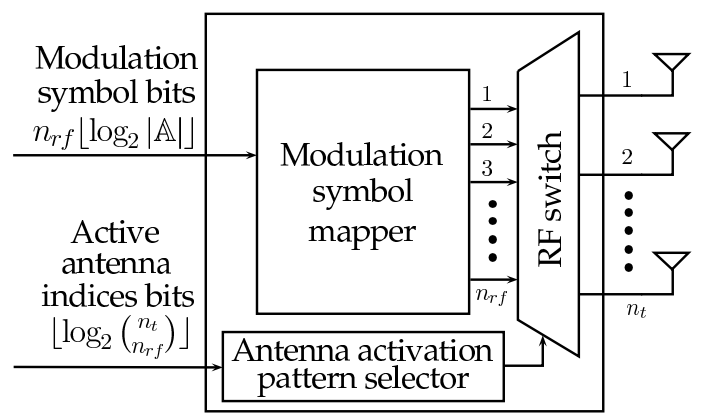

Fig. 1. GSM transmitter.

use a lower-order QAM alphabet which is more power efficient.

- We carry out a study of the proposed and existing algorithms, which includes an assessment of their performance and computational complexity. Simulation results show that the proposed detection algorithms have lesser complexity than minimum mean square error (MMSE) detection complexity, while achieving significantly better performance than MMSE detection performance.

The rest of the paper is organized as follows. The multiuser GSM-MIMO system model on the uplink is presented in Section [I In Section [II, we derive an analytical upper bound on the ML detection performance in multiuser GSM-MIMO. In Section IV we present the proposed detection and channel estimation algorithms for multiuser GSM-MIMO and their performance. In Section $\nabla$ the performance of the proposed algorithms in frequency-selective fading are presented. Conclusions are presented in Section VI,

\section{Multiuser GSM-MiMO SYSTEM MODEL}

Consider a multiuser system with $K$ uplink users communicating with a BS having $N$ receive antennas, where $N$ is in the order of tens to hundreds. The ratio $K / N$ is the system loading factor. Users employ GSM for their transmission. Each user has $n_{t}$ transmit antennas and $n_{r f}, 1 \leq n_{r f} \leq n_{t}$, transmit RF chains. An $n_{r f} \times n_{t}$ switch connects the RF chains to the transmit antennas. In a given channel use, each user selects $n_{r f}$ of its $n_{t}$ transmit antennas, and transmits $n_{r f}$ symbols from a modulation alphabet $\mathbb{A}$ on the selected antennas. The remaining $n_{t}-n_{r f}$ antennas remain silent (i.e., they can be viewed as transmitting the value zero). The GSM transmitter at the user terminal is shown in Fig. 11 The selection of $n_{r f}$ active antennas is made based on $\left\lfloor\log _{2}\left(\begin{array}{c}n_{t} \\ n_{r f}\end{array}\right)\right\rfloor$ information bits. For example, for $n_{t}=4$ and $n_{r f}=2$, two out of the four antennas are selected using $\left\lfloor\log _{2}\left(\begin{array}{c}4 \\ 2\end{array}\right)\right\rfloor=\left\lfloor\log _{2} 6\right\rfloor=\lfloor 2.585\rfloor=2$ information bits. The mapping of information bits to active antenna indices in GSM is described below.

Define an 'antenna activation pattern' to be an $n_{t} \times 1$ vector consisting of 1's and 0's, where a 1 in a coordinate indicates that the antenna corresponding to that coordinate is active and a 0 indicates that the corresponding antenna is silent. Note that there are $\left(\begin{array}{c}n_{t} \\ n_{r f}\end{array}\right)$ activation patterns possible. For example, for $n_{t}=4$ and $n_{r f}=2$, the following six activation patterns are possible:

$\left[\begin{array}{llll}1 & 1 & 0 & 0\end{array}\right]^{T},\left[\begin{array}{llll}0 & 0 & 1 & 1\end{array}\right]^{T},\left[\begin{array}{llll}1 & 0 & 1 & 0\end{array}\right]^{T},\left[\begin{array}{llll}0 & 1 & 0 & 1\end{array}\right]^{T},\left[\begin{array}{llll}1 & 0 & 0 & 1\end{array}\right]^{T},\left[\begin{array}{llll}0 & 1 & 1 & 0\end{array}\right]^{T}$. 
Out of the $\left(\begin{array}{c}n_{t} \\ n_{r f}\end{array}\right)$ possible activation patterns, only $2^{\left\lfloor\log _{2}\left(\begin{array}{c}n_{t} \\ n_{r f}\end{array}\right)\right\rfloor}$ activation patterns are needed for signaling. Let $\mathcal{S}$ denote the set of these $2^{\left\lfloor\log _{2}\left(\begin{array}{c}n_{t} \\ n_{r f}\end{array}\right)\right\rfloor}$ activation patterns chosen from the set of all possible patterns, i.e., $|\mathcal{S}|=2^{\left.\log _{2}\left(\begin{array}{c}n_{t} \\ n_{r f}\end{array}\right)\right\rfloor}$. In the above example, let the set of chosen activation patterns be

$$
\mathcal{S}=\left\{\left[\begin{array}{llll}
1 & 1 & 0 & 0
\end{array}\right]^{T},\left[\begin{array}{llll}
1 & 0 & 1 & 0
\end{array}\right]^{T},\left[\begin{array}{llll}
1 & 0 & 0 & 1
\end{array}\right]^{T},\left[\begin{array}{llll}
0 & 1 & 1 & 0
\end{array}\right]^{T}\right\} .
$$

A mapping is done between combinations of $\left\lfloor\log _{2}\left(\begin{array}{c}n_{t} \\ n_{r f}\end{array}\right)\right\rfloor$ information bits to activation patterns in $\mathcal{S}$. The following table shows such a mapping for the $n_{t}=4, n_{r f}=2$ example:

\begin{tabular}{|c|c|c|}
\hline Information bits & Antenna activation pattern & Remarks \\
\hline \hline 00 & {$\left[\begin{array}{llll}1 & 1 & 0 & 0\end{array}\right]^{T}$} & $\begin{array}{c}\text { antennas 1,2: Active; } \\
\text { antennas 3,4: Silent }\end{array}$ \\
\hline 01 & {$\left[\begin{array}{llll}1 & 0 & 1 & 0\end{array}\right]^{T}$} & $\begin{array}{c}\text { antennas 1,3: Active; } \\
\text { antennas 2,4: Silent }\end{array}$ \\
\hline 10 & {$\left[\begin{array}{llll}1 & 0 & 0 & 1\end{array}\right]^{T}$} & $\begin{array}{c}\text { antennas 1,4: Active; } \\
\text { antennas 2,3: Silent }\end{array}$ \\
\hline 11 & {$\left[\begin{array}{llll}0 & 1 & 1 & 0\end{array}\right]^{T}$} & $\begin{array}{c}\text { antennas 2,3: Active; } \\
\text { antennas 1,4: Silent }\end{array}$ \\
\hline
\end{tabular}

TABLE I

MAPPING BETWEEN INFORMATION BITS AND ACTIVE ANTENNA INDICES IN GSM FOR $n_{t}=4, n_{r f}=2$.

Note that the mapping does not need channel state information. Also, the mapping rule between information bits and active antenna indices is made known to the transmitter and receiver a priori for encoding and decoding purposes, respectively.

Apart from the bits conveyed through active antenna indices, additional bits are conveyed through modulation symbols sent through the $n_{r f}$ active antennas. Therefore, the total number of bits conveyed by a GSM transmitter per channel use is given by

$$
\left\lfloor\log _{2}\left(\begin{array}{c}
n_{t} \\
n_{r f}
\end{array}\right)\right\rfloor+n_{r f}\left\lfloor\log _{2}|\mathbb{A}|\right\rfloor \quad \text { bpcu. }
$$

For example, a GSM transmitter with $n_{t}=4, n_{r f}=2$ and 4-QAM conveys 6 bpcu.

GSM signal set: Let $\mathbb{S}_{n_{t}, \mathbb{A}}^{n_{r f}}$ denote the GSM signal set, which is the set of GSM signal vectors that can be transmitted. Then, $\mathbb{S}_{n_{t}, \mathbb{A}}^{n_{r f}}$ is given by

$$
\mathbb{S}_{n_{t}, \mathbb{A}}^{n_{r f}}=\left\{\mathbf{s}: s_{j} \in \mathbb{A} \cup\{0\},\|\mathbf{s}\|_{0}=n_{r f}, \mathcal{I}(\mathbf{s}) \in \mathcal{S}\right\},
$$

where $\mathbf{s}$ is the $n_{t} \times 1$ transmit vector, $s_{j}$ is the $j$ th entry of $\mathbf{s}$, $j=1, \cdots, n_{t},\|\mathbf{s}\|_{0}$ is the $l_{0}$-norm of the vector $\mathbf{s}$, and $\mathcal{I}(\mathbf{s})$ is a function that gives the activation pattern for $\mathbf{s}$; for example, $\mathcal{I}\left(\mathbf{s}=\left[\begin{array}{llll}+1 & -1 & -1 & 0\end{array}\right]^{T}\right)=\left[\begin{array}{llll}1 & 1 & 1 & 0\end{array}\right]^{T}$.

Example: Let $n_{t}=4, n_{r f}=2$, BPSK modulation, and $\mathcal{S}=\left\{\left[\begin{array}{llll}1 & 1 & 0 & 0\end{array}\right]^{T},\left[\begin{array}{llll}1 & 0 & 1 & 0\end{array}\right]^{T},\left[\begin{array}{llll}1 & 0 & 0 & 1\end{array}\right]^{T},\left[\begin{array}{llll}0 & 1 & 1 & 0\end{array}\right]^{T}\right\}$. The GSM signal set for these parameters is given by

$$
\begin{aligned}
& \mathbb{S}_{4, \mathrm{BPSK}}^{2}=\left\{\left[\begin{array}{c}
+1 \\
+1 \\
0 \\
0
\end{array}\right],\left[\begin{array}{c}
+1 \\
-1 \\
0 \\
0
\end{array}\right],\left[\begin{array}{c}
-1 \\
-1 \\
0 \\
0
\end{array}\right],\left[\begin{array}{c}
-1 \\
+1 \\
0 \\
0
\end{array}\right],\left[\begin{array}{c}
+1 \\
0 \\
+1 \\
0
\end{array}\right],\left[\begin{array}{c}
+1 \\
0 \\
-1 \\
0
\end{array}\right],\left[\begin{array}{c}
-1 \\
0 \\
-1 \\
0
\end{array}\right],\left[\begin{array}{c}
-1 \\
0 \\
+1 \\
0
\end{array}\right],\right. \\
& \left.\left[\begin{array}{c}
+1 \\
0 \\
0 \\
+1
\end{array}\right],\left[\begin{array}{c}
+1 \\
0 \\
0 \\
-1
\end{array}\right],\left[\begin{array}{c}
-1 \\
0 \\
0 \\
-1
\end{array}\right],\left[\begin{array}{c}
-1 \\
0 \\
0 \\
+1
\end{array}\right],\left[\begin{array}{c}
0 \\
+1 \\
+1 \\
0
\end{array}\right],\left[\begin{array}{c}
0 \\
+1 \\
-1 \\
0
\end{array}\right],\left[\begin{array}{c}
0 \\
-1 \\
-1 \\
0
\end{array}\right],\left[\begin{array}{c}
0 \\
-1 \\
+1 \\
0
\end{array}\right]\right\} \text {. }
\end{aligned}
$$

Figures 2(a) and 2(b) illustrate large-scale multiuser GSMMIMO system and conventional multiuser MIMO (massive
MIMO) system, respectively. Let $\mathbf{x}_{k} \in \mathbb{S}_{n_{t}, \mathbb{A}}^{n_{r f}}$ denote the trans-

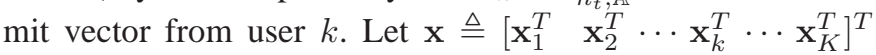
denote the vector comprising of transmit vectors from all the users, where $(.)^{T}$ denotes transpose operation. Note that $\mathbf{x} \in\left(\mathbb{S}_{n_{t}, \mathbb{A}}^{n_{r f}}\right)^{K}$.

Let $\mathbf{H} \in \mathbb{C}^{N \times K n_{t}}$ denote the channel gain matrix, where $H_{i,(k-1) n_{t}+j}$ denotes the complex channel gain from the $j$ th transmit antenna of the $k$ th user to the $i$ th BS receive antenna. The channel gains are assumed to be independent Gaussian with zero mean and variance $\sigma_{\kappa}^{2}$, such that $\sum_{\kappa=1}^{K n_{t}} \sigma_{\kappa}^{2}=K n_{t}$. The $\sigma_{\kappa}^{2}$ models the imbalance in the received power from the $\kappa$ th antenna, $\kappa \in\left\{1, \cdots, K n_{t}\right\}$, due to path loss etc., and $\sigma_{\kappa}^{2}=1$ corresponds to the case of perfect power control. Assuming perfect synchronization, the received signal at the $i$ th BS antenna is given by

$$
y_{i}=\sum_{k=1}^{K} \mathbf{h}_{i,[k]} \mathbf{x}_{k}+n_{i},
$$

where $\mathbf{h}_{i,[k]}$ is a $1 \times n_{t}$ vector obtained from the $i$ th row of $\mathbf{H}$ and $(k-1) n_{t}+1$ to $k n_{t}$ columns of $\mathbf{H}$, and $n_{i}$ is the noise modeled as a complex Gaussian random variable with zero mean and variance $\sigma^{2}$. The received signal at the BS antennas can be written in vector form as

$$
\mathbf{y}=\mathbf{H x}+\mathbf{n},
$$

where $\mathbf{y}=\left[y_{1}, y_{2}, \cdots, y_{N}\right]^{T}$ and $\mathbf{n}=\left[n_{1}, n_{2}, \cdots, n_{N}\right]^{T}$. For this system model, the ML detection rule is given by

$$
\hat{\mathbf{x}}=\underset{\mathbf{x} \in\left(\mathbb{S}_{n_{t}, \mathbb{A}}^{n_{r}}\right) K}{\operatorname{argmin}}\|\mathbf{y}-\mathbf{H} \mathbf{x}\|^{2},
$$

where $\|\mathbf{y}-\mathbf{H x}\|^{2}$ is the ML cost. The maximum a posteriori probability (MAP) decision rule is given by

$$
\hat{\mathbf{x}}=\underset{\mathbf{x} \in\left(\mathbb{S}_{n_{t}, \mathbb{A}}^{n_{r f}}\right)^{K}}{\operatorname{argmax}} \operatorname{Pr}(\mathbf{x} \mid \mathbf{y}, \mathbf{H}) .
$$

Since $\left|\left(\mathbb{S}_{n_{t}, \mathbb{A}}^{n_{r f}}\right)^{K}\right|=\left(|\mathcal{S}||\mathbb{A}|^{n_{r f}}\right)^{K}$, the exact computation of (5) and (6) requires exponential complexity in $K$. In the next section, we derive an analytical upper bound on the ABEP for ML detection. In Section IV we propose message passing based detection algorithms which give approximate solutions to (6) at low complexities.

We note that the condition for the spectral efficiencies of GSM-MIMO (with $n_{t}$ transmit antennas, $n_{r f}$ transmit RF chains, and modulation alphabet $\mathbb{A}$ at each user) and conventional multiuser MIMO (with $m_{t}$ transmit antennas, $m_{t}$ transmit RF chains, and modulation alphabet $\mathbb{B}$ at each user) to be the same is given by

$$
|\mathcal{S}||\mathbb{A}|^{n_{r f}}=|\mathbb{B}|^{m_{t}} .
$$

For example, (i) GSM-MIMO with BPSK, $n_{t}=4, n_{r f}=2$, (ii) SM-MIMO with 4-QAM, $n_{t}=4, n_{r f}=1$, and (iii) conventional multiuser MIMO with 4-QAM, 2 transmit antennas and 2 transmit RF chains, all have the same spectral efficiency of 4 bpcu per user. In conventional multiuser MIMO, the vector $\mathbf{x} \in \mathbb{B}^{K m_{t}}$ and the channel matrix $\mathbf{H} \in \mathbb{C}^{N \times K m_{t}}$. 


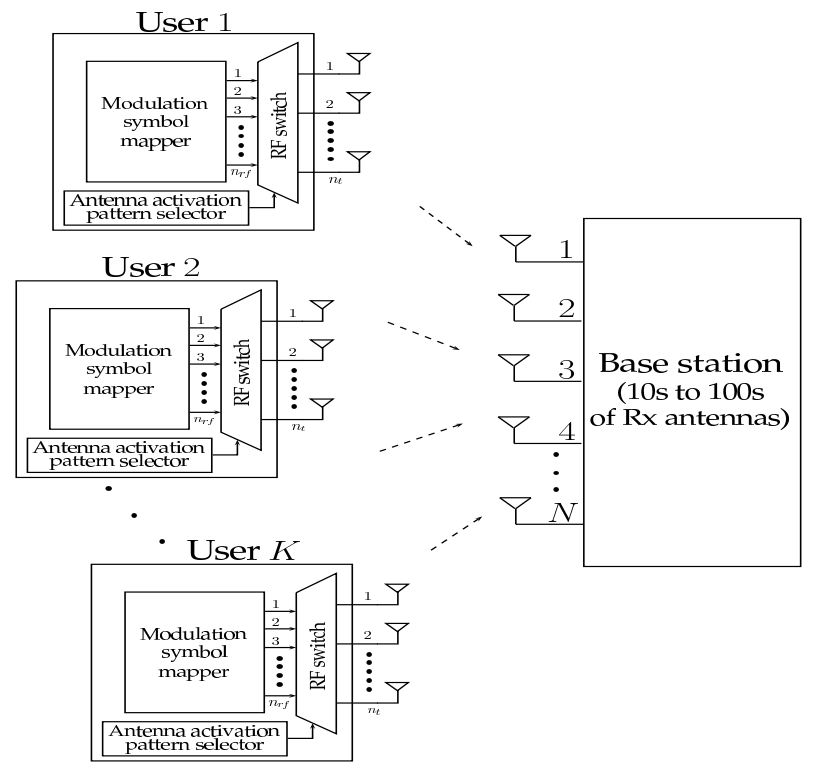

(a) Multiuser GSM-MIMO system.
User 1

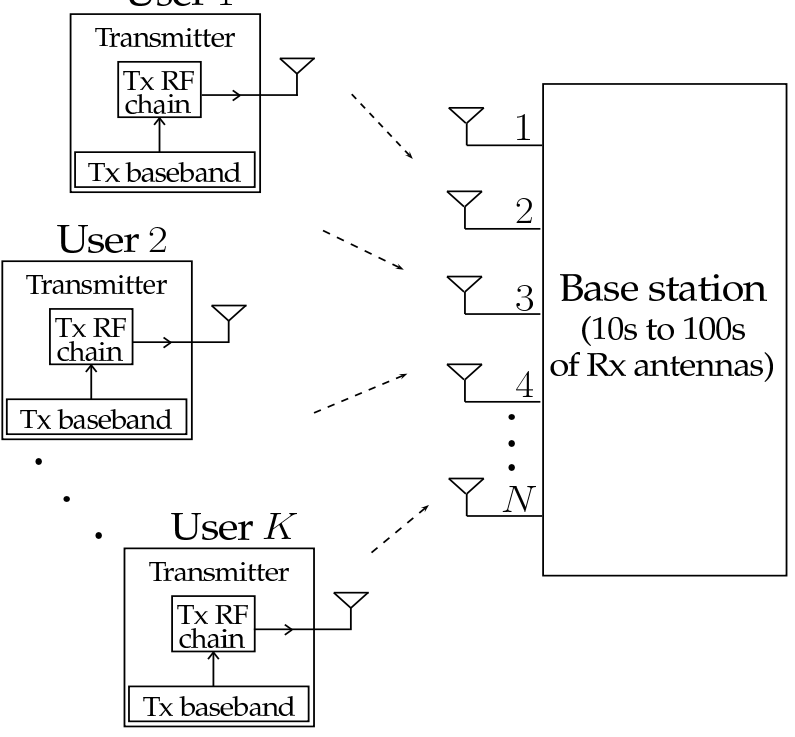

(b) Conventional multiuser MIMO system.

Fig. 2. Large-scale multiuser GSM-MIMO and conventional multiuser MIMO system architectures.

\section{AVERAGE BEP ANALYSis}

In this section, we analyze the average BEP performance of ML detection in multiuser GSM-MIMO. Assume that all the transmit GSM signal vectors are equally likely. The ML detection rule in (5) can be written as

$$
\hat{\mathbf{x}}=\underset{\mathbf{x} \in \mathbb{G}}{\operatorname{argmin}}\left\|\mathbf{y}-\sum_{\kappa=1}^{K n_{t}} x_{\kappa} \mathbf{h}_{\kappa}\right\|^{2},
$$

where $x_{\kappa}$ is the $\kappa$ th element of $\mathbf{x}, \mathbf{h}_{\kappa}$ is the $\kappa$ th column of $\mathbf{H}$ and $\mathbb{G} \triangleq\left(\mathbb{S}_{n_{t}, \mathbb{A}}^{n_{r f}}\right)^{K}$. The pairwise error probability (PEP) of $\mathbf{x}$ being decoded as $\tilde{\mathbf{x}} \in \mathbb{G}$ can be written as

$$
\begin{array}{r}
P(\mathbf{x} \rightarrow \tilde{\mathbf{x}} \mid \mathbf{H})=P\left(\left\|\mathbf{y}-\sum_{\kappa=1}^{K n_{t}} x_{\kappa} \mathbf{h}_{\kappa}\right\|^{2}>\left\|\mathbf{y}-\sum_{\kappa=1}^{K n_{t}} \tilde{x}_{\kappa} \mathbf{h}_{\kappa}\right\|^{2} \mid \mathbf{H}\right) \\
=P\left(\sum_{i=1}^{N}\left|y_{i}-\sum_{\kappa=1}^{K n_{t}} x_{\kappa} h_{i, \kappa}\right|^{2}>\sum_{i=1}^{N}\left|y_{i}-\sum_{\kappa=1}^{K n_{t}} \tilde{x}_{\kappa} h_{i, \kappa}\right|^{2} \mid \mathbf{H}\right),
\end{array}
$$

where $h_{i, \kappa}$ is the $i$ th element of $\mathbf{h}_{\kappa}$. Let $A_{i} \triangleq \sum_{\kappa=1}^{K n_{t}} x_{\kappa} h_{i, \kappa}$ and $\tilde{A}_{i} \triangleq \sum_{\kappa=1}^{K n_{t}} \tilde{x}_{\kappa} h_{i, \kappa}$. Since $\mathbf{x}$ is the transmitted vector, $y_{i}=A_{i}+n_{i}, i=1, \cdots, N$. Now, we can write

$$
\begin{aligned}
P(\mathbf{x} \rightarrow \tilde{\mathbf{x}} \mid \mathbf{H}) & =P\left(\sum_{i=1}^{N}\left|y_{i}-A_{i}\right|^{2}>\sum_{i=1}^{N}\left|y_{i}-\tilde{A}_{i}\right|^{2} \mid \mathbf{H}\right) \\
& =P\left(\sum_{i=1}^{N}\left|n_{i}\right|^{2}>\sum_{i=1}^{N}\left|A_{i}+n_{i}-\tilde{A}_{i}\right|^{2} \mid \mathbf{H}\right) \\
& =P\left(\sum_{i=1}^{N} 2 \Re\left(\left(\tilde{A}_{i}-A_{i}\right) n_{i}^{*}\right)>\sum_{i=1}^{N}\left|A_{i}-\tilde{A}_{i}\right|^{2} \mid \mathbf{H}\right)
\end{aligned}
$$

where $\Re($.$) denotes real part, (.)* denotes conjugation, and$ $\sum_{i=1}^{N} 2 \Re\left(\left(\tilde{A}_{i}-A_{i}\right) n_{i}^{*}\right)$ is a Gaussian random variable with mean zero and variance $2 \sigma^{2} \sum_{i=1}^{N}\left|A_{i}-\tilde{A}_{i}\right|^{2}$. Therefore,

$$
\begin{aligned}
P(\mathbf{x} \rightarrow \tilde{\mathbf{x}} \mid \mathbf{H}) & =Q\left(\sqrt{\sum_{i=1}^{N}\left|A_{i}-\tilde{A}_{i}\right|^{2} / 2 \sigma^{2}}\right) \\
& =Q\left(\sqrt{\left\|\sum_{\kappa=1}^{K n_{t}}\left(x_{\kappa}-\tilde{x}_{\kappa}\right) \mathbf{h}_{\kappa}\right\|^{2} / 2 \sigma^{2}}\right) .
\end{aligned}
$$

The argument in (10) has a central $\chi^{2}$-distribution with $2 N$ degrees of freedom. Computation of the unconditional PEP $P(\mathbf{x} \rightarrow \tilde{\mathbf{x}})$ requires the expectation of the $Q($.$) function in$ (10) w.r.t. H, which can be obtained as follows [21]:

$$
\begin{aligned}
P(\mathbf{x} \rightarrow \tilde{\mathbf{x}}) & =\mathbb{E}_{\mathbf{H}}\{P(\mathbf{x} \rightarrow \tilde{\mathbf{x}} \mid \mathbf{H})\} \\
& =f(\alpha)^{N} \sum_{i=0}^{N-1}\left(\begin{array}{c}
N-1+i \\
i
\end{array}\right)(1-f(\alpha))^{i},
\end{aligned}
$$

where $f(\alpha) \triangleq \frac{1}{2}\left(1-\sqrt{\frac{\alpha}{1+\alpha}}\right), \alpha \triangleq \frac{1}{4 \sigma^{2}} \sum_{\kappa=1}^{K n_{t}} \theta_{\kappa}$, and $\theta_{\kappa} \triangleq$ $\left|x_{\kappa}-\tilde{x}_{\kappa}\right|^{2}$. Now, an upper bound on the average BEP based on union bounding can be obtained as

$$
P_{B} \leq \frac{1}{2^{\eta}} \sum_{\mathbf{x} \in \mathbb{G}} \sum_{\tilde{\mathbf{x}} \in \mathbb{G} \backslash \mathbf{x}} P(\mathbf{x} \rightarrow \tilde{\mathbf{x}}) \frac{d(\mathbf{x}, \tilde{\mathbf{x}})}{\eta},
$$

where $d(\mathbf{x}, \tilde{\mathbf{x}})$ is the number of bits in which $\mathbf{x}$ differs from $\tilde{\mathbf{x}}$. The total number of PEPs to be calculated in (12) is $2^{\eta}\left(2^{\eta}-1\right)$. Therefore, the complexity of the computation of the bound in (12) will increase exponentially in $K, n_{t}$, and $n_{r f}$. In the following subsection, we devise simplification methods to reduce this computational complexity.

\section{A. Reduction of computation complexity in (12)}

The expression for $P_{B}$ in (12) can be written in the following form:

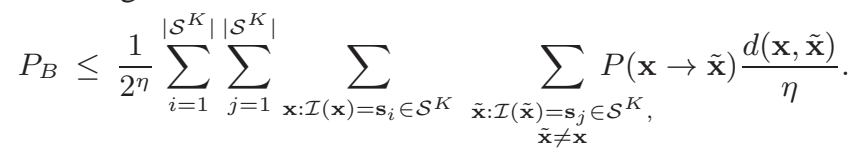


For a given pair of antenna activation patterns $\mathbf{s}_{i}$ and $\mathbf{s}_{j}, i, j \in$ $\left\{1, \cdots,|\mathcal{S}|^{K}\right\}$, the total number of PEPs are $|\mathbb{A}|^{2 K n_{r f}}$ when $i \neq j$, and $|\mathbb{A}|^{K n_{r f}}\left(|\mathbb{A}|^{K n_{r f}}-1\right)$ when $i=j$.

Complexity reduction 1: For a pair of activation patterns $\mathbf{s}_{i}$ and $\mathbf{s}_{j}$, let $\mathcal{A}_{i j}$ denote the set of active antennas that are common to both $\mathbf{s}_{i}$ and $\mathbf{s}_{j}$. Define $\beta_{i j}=K n_{r f}-\left|\mathcal{A}_{i j}\right|$. Note that $\beta_{i j} \in\left\{0,1, \cdots, \min \left(n_{r f}, n_{t}-n_{r f}\right) K\right\}$. Also, note that for any $i, j$ for which $\beta_{i j}=q$, the value of the summation $\sum_{\mathbf{x}: \mathcal{I}(\mathbf{x})=\mathbf{s}_{i}} \sum_{\tilde{\mathbf{x}}: \mathcal{I}(\tilde{\mathbf{x}})=\mathbf{s}_{j}, \tilde{\mathbf{x}} \neq \mathbf{x}} P(\mathbf{x} \rightarrow \tilde{\mathbf{x}})$ in 13 will be the same, and so it is enough to compute this summation only once for each $q$. With this simplification, 13) can be written as

$$
P_{B} \leq \frac{1}{2^{\eta}} \sum_{q=0}^{\min \left(n_{r f}, n_{t}-n_{r f}\right) K} \phi(q) \sum_{\mathbf{x}: \mathcal{I}(\mathbf{x})=\mathbf{s}_{i}} \sum_{\substack{\tilde{\mathbf{x}}: \mathcal{I}(\tilde{\mathbf{x}})=\mathbf{s}_{j} \\ \beta_{i j}=q}} P(\mathbf{x} \rightarrow \tilde{\mathbf{x}}) \frac{d(\mathbf{x}, \tilde{\mathbf{x}})}{\eta},
$$

where $\phi(q)$ is the number of $\left(\mathbf{s}_{i}, \mathbf{s}_{j}\right)$ pairs for which $\beta_{i j}=q$, which can be computed easily.

Example: A direct computation of the first two summations in (13) is prohibitive, as the total number of terms is exponential in $K, n_{t}, n_{r f}$. For $K=2, n_{t}=4$ and $n_{r f}=2,\left|\mathcal{S}^{K}\right|=2^{4}$. So, the first two summations in 13. will have $2^{8}=256$ terms. Whereas for these parameters, $q \in\{0,1,2,3,4\}, \phi(\{0,1,2,3,4\})=\{16,88,128,22,2\}$ and $\sum_{q=0}^{4} \phi(q)=256$. Hence, the inner summations can be computed only 5 times (once for each $q$ ), instead of 256 times.

Complexity reduction 2: For each value of $q$, we need to compute $|\mathbb{A}|^{2 K n_{r f}}$ PEPs. We propose to reduce this complexity as follows. The parameter $\alpha$ in 111 is the summation of $K n_{t}$ terms. Out of these $K n_{t}$ terms, $K n_{t}-\left(K n_{r f}+q\right)$ terms will be zero for a given value of $q$. Of the $\left(K n_{r f}+q\right)$ non-zero terms, $2 q$ terms will take values from $\mathbb{J} \triangleq\left\{|c|^{2}: c \in \mathbb{A}\right\}$, and $K n_{r f}-q$ terms will take values from $\mathbb{L} \triangleq\left\{|c-\tilde{c}|^{2}: c, \tilde{c} \in \mathbb{A}\right\}$. Let $\mathbb{J}=\left\{j_{1}, j_{2}, \cdots, j_{m}\right\}$ and $\mathbb{L}=\left\{l_{1}, l_{2}, \cdots, l_{n}\right\}$, where $j_{1}<j_{2}<\cdots<j_{m}, l_{1}<l_{2}<\cdots<l_{n}, m=|\mathbb{J}|$, and $n=|\mathbb{L}|$. We write $\alpha$ as $\alpha=\alpha_{1}+\alpha_{2}$, where $\alpha_{1}$ is the sum of $2 q$ terms from $\mathbb{J}$ and $\alpha_{2}$ is the sum of $K n_{r f}-q$ terms from $\mathbb{L}$. Note that $\alpha_{1}$ can take values in the range $2 q j_{1}$ to $2 q j_{m}$. For a given value of $\alpha_{1}$, the following equations must be satisfied:

$$
\sum_{i=1}^{m} j_{i} v_{i}=\alpha_{1}, \quad \sum_{i=1}^{m} v_{i}=2 q
$$

where $v_{i}$ is an integer such that $v_{i} \in\left\{0,1, \cdots,\left\lfloor\left(\alpha_{1}-\right.\right.\right.$ $\left.\left.\left.\sum_{k=i+1}^{m} j_{k} v_{k}\right) / j_{i}\right\rfloor\right\}$. Similarly, $\alpha_{2}$ can take values in the range $\left(K n_{r f}-q\right) l_{1}$ to $\left(K n_{r f}-q\right) l_{n}$, and, for a given value of $\alpha_{2}$, the following equations must be satisfied:

$$
\sum_{i=1}^{n} l_{i} u_{i}=\alpha_{2}, \quad \sum_{i=1}^{n} u_{i}=K n_{r f}-q,
$$

where $u_{i}$ is an integer such that $u_{i} \in\left\{0,1, \cdots,\left\lfloor\left(\alpha_{2}-\right.\right.\right.$ $\left.\left.\left.\sum_{k=i+1}^{n} l_{k} u_{k}\right) / l_{i}\right\rfloor\right\}$. Since $\alpha=\alpha_{1}+\alpha_{2}, \alpha$ lies in the range $2 q j_{1}+\left(K n_{r f}-q\right) l_{1}$ to $2 q j_{m}+\left(K n_{r f}-q\right) l_{n}$. A given value of $\alpha$ can be written as

$\alpha=\sum_{i=1}^{m} j_{i} v_{i}+\sum_{i=1}^{n} l_{i} u_{i} \quad$ s.t. $\sum_{i=1}^{m} v_{i}=2 q, \sum_{i=1}^{n} u_{i}=K n_{r f}-q$.

The choices of $v_{i}$ 's and $u_{i}$ 's to attain a particular $\alpha$ is not unique, i.e., there exist multiple pairs of $\mathbf{x}$ and $\tilde{\mathbf{x}}$ that

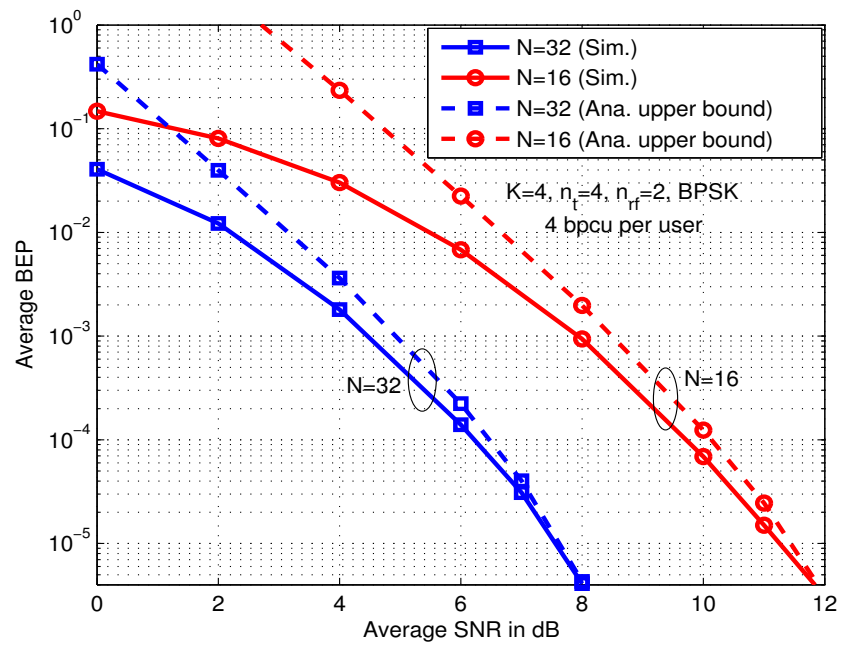

Fig. 3. Comparison between analytical ABEP upper bound and simulated ABEP for ML detection in GSM-MIMO with $N=16,32, K=4, n_{t}=4$, $n_{r f}=2$, BPSK, and 4 bpcu per user. Analysis and simulation.

correspond to different values of $v_{i}$ 's and $u_{i}$ 's but the same value of $\alpha$. Thus, we need to evaluate (11) only once for a given $\alpha$ and count the number of possible combinations of $v_{i}$ 's and $u_{i}$ 's that correspond to that $\alpha$.

Example: When $n_{t}=4, n_{r f}=3, \mathbb{A}=\{-1-\mathbf{j},-1+\mathbf{j}, 1-$ $\mathbf{j}, 1+\mathbf{j}\}$, where $\mathbf{j}=\sqrt{-1}$, then, $\mathbb{J}=\{2\}, \mathbb{L}=\{0,4,8\}$. For a particular value of $q$, say $q=1$, the summation in (14) requires computation of PEP for 64 different pairs of GSM signals. But $\alpha$ lies in the range 2 to 18 , and hence we need to compute only 17 PEP terms.

\section{B. Numerical results}

In Fig. 3, we compare the analytical ABEP upper bound and the simulated ABEP of multiuser GSM-MIMO with ML detection for the following system parameter settings: $N=$ $16,32, K=4, n_{t}=4, n_{r f}=2$, BPSK, and 4 bpcu per user. It can be observed that the upper bound is tight at moderate to high SNRs. It is also observed that, as expected, both analysis and simulation predict that the ABEP performance improves as the number of BS antennas $N$ is increased.

In Fig. 4, we compare the ABEP performance of the following four different systems with $N=8$ and $K=2$ : System 1 - conventional multiuser MIMO with $n_{t}=n_{r f}=1$, 16-QAM; System 2 - conventional multiuser MIMO with $n_{t}=n_{r f}=2$, 8-QAM; System 3 - multiuser SM-MIMO with $n_{t}=4, n_{r f}=1$, 16-QAM; and System 4 - multiuser GSM-MIMO with $n_{t}=4, n_{r f}=2$, 4-QAM. Note that all the four systems achieve the same spectral efficiency of $6 \mathrm{bpcu}$ per user. The first two systems are conventional multiuser MIMO systems where $n_{t}=n_{r f}$. System 1 uses one transmit antenna and one transmit RF chain at each user and achieves 6 bpcu per user by using 64-QAM. On the other hand, System 2 uses two transmit antennas and two transmit RF chains at each user and achieves 6 bpcu per user by using 8-QAM. System 3 is a multiuser SM-MIMO system where each user uses four transmit antennas but only one transmit RF chain. Each user in this system uses 16-QAM to achieve 6 bpcu per user; 4 bits through 16-QAM and 2 bits through indexing. System 4 


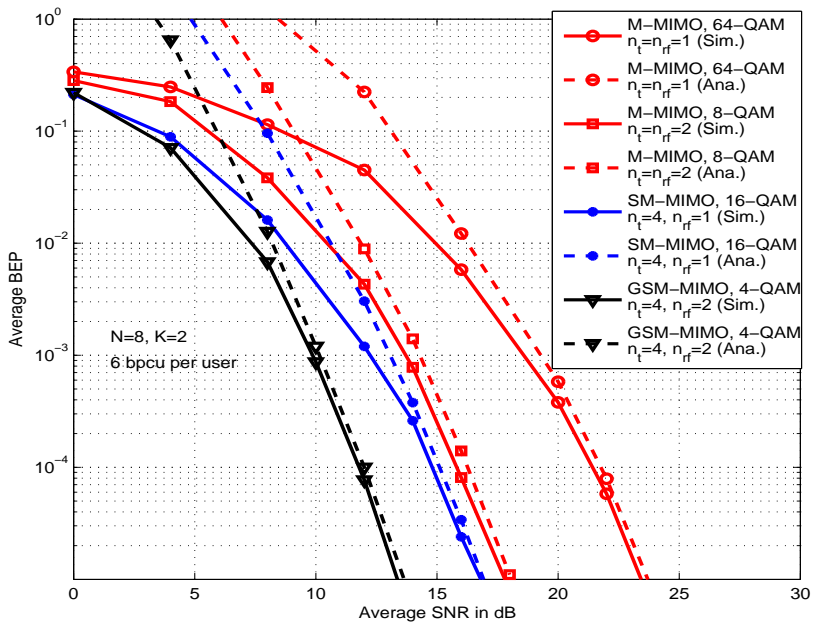

Fig. 4. Comparison between the ABEP performance of four different systems, all with $N=8, K=2$, and 6 bpcu per user: (i) conventional multiuser MIMO, $n_{t}=n_{r f}=1$, 64-QAM; ( $\left.i i\right)$ conventional multiuser MIMO, $n_{t}=$ $n_{r f}=2$, 8-QAM; (iii) multiuser SM-MIMO, $n_{t}=4, n_{r f}=1$, 16-QAM; and (iv) multiuser GSM-MIMO, $n_{t}=4, n_{r f}=2$, 4-QAM. Analysis and simulation.

is a GSM-MIMO system where each user uses four transmit antennas and two transmit RF chains. This system uses 4QAM on two streams to achieve 6 bpcu per user; four bits through modulation symbols (i.e., two 4-QAM symbols on two streams) and 2 bits through indexing.

The following observations can be made from Fig. 4. First, it can be observed that multiuser SM-MIMO system (System 3) achieves better performance compared to conventional multiuser MIMO systems (Systems 1 and 2) by about 2 to 7 $\mathrm{dB}$ at $10^{-3}$ BER. The better performance of SM-MIMO over conventional MIMO in System 1 can be attributed to the fact that, to achieve the same spectral efficiency of 6 bpcu per user, SM-MIMO can use a smaller-sized QAM alphabet (16-QAM) than that used in system 1 (64-QAM), and that a smaller-sized QAM alphabet is more power efficient than a larger-sized one. Also, the better performance of SM-MIMO over conventional MIMO in system 2 is attributed to the fact that, although System 2 uses a smaller-sized alphabet (8-QAM) than that in SM-MIMO (16-QAM), there is interference from multiple spatial streams in System 2. Another observation in Fig. 4 is that multiuser GSM-MIMO system (System 4) performs better than multiuser SM-MIMO system (System 3) as well as conventional MIMO system in System 2 which also uses two RF chains like GSM-MIMO. This is because, though GSMMIMO uses two spatial streams like conventional MIMO, its alphabet size is smaller than that in conventional MIMO.

\section{LOW-COMPLEXITY ReCEIVER AlgorithmS}

Optimal detection of multiuser GSM-MIMO signals in large dimensions is prohibitively complex. Generalized sphere decoding (GSD) approach [19], [20] can be employed for GSMMIMO detection. But the complexity of GSD can be high (exponential complexity at low-to-medium SNRs). Therefore, for large systems, GSD becomes prohibitively complex. In this section, we present low-complexity near-optimal detection and channel estimation algorithms for large-scale multiuser GSMMIMO systems. Two approximate message passing based algorithms for detection are presented. In the first algorithm, abbreviated as MP-GSM (message passing for GSM) detection algorithm, messages are formed based on the basic multiuser GSM-MIMO system model in (4). In the second algorithm, abbreviated as CHEMP-GSM (channel hardening-exploiting message passing for GSM) algorithm, messages are formed based on a matched filtered version of the basic system model in (4). We also present a channel estimation approach that directly obtains an estimate of $\mathbf{H}^{H} \mathbf{H}$ for use in the CHEMPGSM algorithm.

\section{A. MP-GSM detection algorithm}

Consider the multiuser GSM-MIMO system model in (4). We model this system as a fully connected factor graph with $K$ variable nodes (or factor nodes) corresponding to $\mathbf{x}_{k}$ 's and $N$ observation nodes corresponding to $y_{i}$ 's, as shown in Fig. 5(a). We aim to get approximate MAP solution through message passing on this graph, where messages are formed by approximating the probability density of the interference as Gaussian.

Messages: The messages passed between variable nodes and observation nodes in the factor graph are derived as follows. Equation (4) can be written as

$$
y_{i}=\mathbf{h}_{i,[k]} \mathbf{x}_{k}+\underbrace{\sum_{j=1, j \neq k}^{K} \mathbf{h}_{i,[j]} \mathbf{x}_{j}+n_{i}}_{\triangleq g_{i k}},
$$

where $\mathbf{h}_{i,[j]}$ is a row vector of length $n_{t}$, given by $\left[H_{i,(j-1) n_{t}+1} \quad H_{i,(j-1) n_{t}+2} \cdots H_{i, j n_{t}}\right]$, and $\mathbf{x}_{j} \in \mathbb{S}_{n_{t}, \mathbb{A}}^{n_{r f}}$. The term $g_{i k}$ defined in (17) is approximated to be a Gaussian random variable 1 with mean $\mu_{i k}$ and variance $\sigma_{i k}^{2}$. The mean $\mu_{i k}$ in the approximation is given by

$$
\begin{aligned}
\mu_{i k} & =\mathbb{E}\left[\sum_{j=1, j \neq k}^{K} \mathbf{h}_{i,[j]} \mathbf{x}_{j}+n_{i}\right] \\
& =\sum_{j=1, j \neq k}^{K} \sum_{\mathbf{s} \in \mathbb{S}_{n_{t}, \mathrm{~A}}^{n_{r f}}} p_{j i}(\mathbf{s}) \mathbf{h}_{i,[j]} \mathbf{S} \\
& =\sum_{j=1, j \neq k}^{K} \sum_{\mathbf{s} \in \mathbb{S}_{n_{t}, \mathbb{A}}^{n_{r f}}} p_{j i}(\mathbf{s}) \sum_{l \in \mathcal{I}(\mathbf{s})} s_{l} H_{i,(j-1) n_{t}+l},
\end{aligned}
$$

where $s_{l}$ 's are the non-zero entries in $\mathbf{s}$ and $l$ 's are their corresponding indices, and the variance $\sigma_{i k}^{2}$ is given by

$$
\begin{aligned}
\sigma_{i k}^{2} & =\operatorname{Var}\left(\sum_{j=1, j \neq k}^{K} \mathbf{h}_{i,[j]} \mathbf{x}_{j}+n_{i}\right) \\
& =\sum_{\substack{j=1 \\
j \neq k}}^{K} \sum_{\mathbf{s} \in \mathbb{S}_{n_{t}, \mathbb{A}}^{n_{r f}}} p_{j i}(\mathbf{s}) \mathbf{h}_{i,[j]} \mathbf{s} \mathbf{s}^{H} \mathbf{h}_{i,[j]}^{H}-\left|\sum_{\mathbf{s} \in \mathbb{S}_{n_{t}, \mathbb{A}}} p_{j i}(\mathbf{s}) \mathbf{h}_{i,[j]} \mathbf{s}\right|^{2}+\sigma^{2},
\end{aligned}
$$

where $(.)^{H}$ denotes conjugate transpose operation, and $p_{k i}(\mathbf{s})$ is the posterior probability given by

$$
p_{k i}(\mathbf{s}) \propto \prod_{m=1, m \neq i}^{N} \exp \left(\frac{-\left|y_{m}-\mu_{m k}-\mathbf{h}_{m,[k]} \mathbf{s}\right|^{2}}{2 \sigma_{m k}^{2}}\right) .
$$

${ }^{1}$ This Gaussian approximation will be accurate for large $K$; example, in systems with tens of users. 


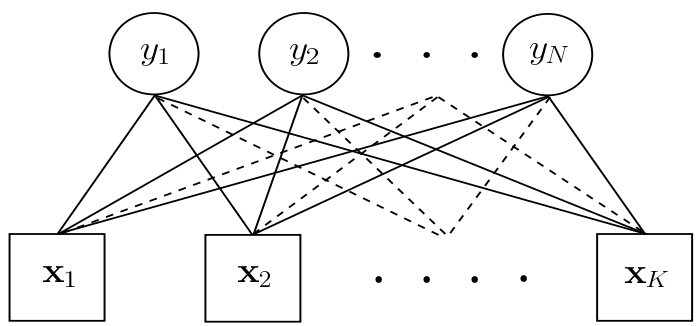

(a) Factor graph

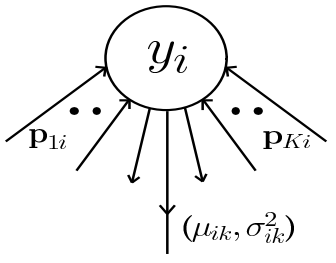

(b) Observation node messages

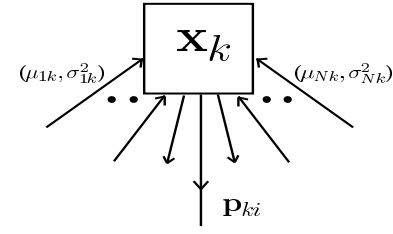

(c) Variable node messages
Fig. 5. The factor graph and messages passed in the MP-GSM algorithm.

Message passing: The messages exchanged between observation and variable nodes are illustrated in Figs. 5(b) and 5(c). The message from observation node $y_{i}$ to variable node $\mathbf{x}_{k}$ consists of the two scalar variables $\mu_{i k}$ and $\sigma_{i k}^{2}$. The message from variable node $\mathbf{x}_{k}$ to observation node $y_{i}$ is a vector message given by $\mathbf{p}_{k i}=\left[p_{k i}\left(\mathbf{s}_{1}\right), p_{k i}\left(\mathbf{s}_{2}\right), \cdots, p_{k i}\left(\mathbf{s}_{\left|\mathbb{S}_{n_{t}, \mathbb{A}}^{n_{r f}}\right|}\right)\right]$. The message passing steps are as follows.

Step 1: Initialize $p_{k i}(\mathbf{s})$ to $1 /\left|\mathbb{S}_{n_{t}, \mathbb{A}}^{n_{r f}}\right|$ for all $i, k$ and $\mathbf{s}$.

Step 2: Compute $\mu_{i k}$ and $\sigma_{i k}^{2}$ from (18) and (19), respectively. Step 3: Compute $p_{k i}$ from 20. To improve the convergence rate, damping 2 of the messages in 201 is done with a damping factor $\delta \in(0,1]$, as shown in the algorithm listing in Algorithm 1. The symbol probabilities at the end of an iteration are computed as

$p_{k}(\mathbf{s}) \propto \prod_{i=1}^{N} \exp \left(\frac{-\left|y_{i}-\mu_{i k}-\mathbf{h}_{i,[k]} \mathbf{s}\right|^{2}}{2 \sigma_{i k}^{2}}\right), k=1, \cdots, K$.

Stopping criterion: Repeat Steps 2 and $\mathbf{3}$ until $\left\|\mathbf{p}^{(t)}-\mathbf{p}^{(t+1)}\right\|<\epsilon$ or until the number of iterations is less than a fixed maximum number, where $\mathbf{p}^{(t)}=$ $\left[p_{1}^{(t)}\left(s_{1}\right), \cdots, p_{1}^{(t)}\left(s_{\left|\mathbb{S}_{n_{t}, \mathbb{A}}^{n_{r f}}\right|}\right), \cdots, p_{K}^{(t)}\left(s_{1}\right), \cdots, p_{K}^{(t)}\left(s_{\left|\mathbb{S}_{n_{t}, \mathbb{A}}^{n_{r f}}\right|}\right)\right]^{T}$ and $\epsilon$ is a pre-fixed constant such that $0<\epsilon \ll 1$.

The detected vector of the $k$ th user at the BS is obtained as

$$
\hat{\mathbf{x}}_{k}=\underset{\mathbf{s} \in \mathbb{S}_{n_{t}, \mathbb{A}}^{n_{r f}}}{\operatorname{argmax}} p_{k}(\mathbf{s}) .
$$

The non-zero entries in $\hat{\mathbf{x}}_{k}$ and their indices are then demapped to obtain the information bits of the $k$ th user. The MP-GSM algorithm listing is given in Algorithm 1.

Complexity and performance of MP-GSM algorithm: Here, we present and discuss the complexity and performance of the MP-GSM detection algorithm.

Complexity: From (18), (19), and 20), we see that the total complexity of the MP-GSM algorithm is $O\left(N K\left|\mathbb{S}_{n_{t}, \mathbb{A}}^{n_{r f}}\right|\right)$. We compare this complexity with that of the detection using the MMSE estimate given by $\left(\mathbf{H}^{H} \mathbf{H}+\frac{1}{S N R} \mathbf{I}\right)^{-1} \mathbf{H}^{H} \mathbf{y}$. The

\footnotetext{
${ }^{2}$ Damping can be thought of as reweighting the messages with a reweighting parameter (damping factor) $\delta[26]-[29]$.
}

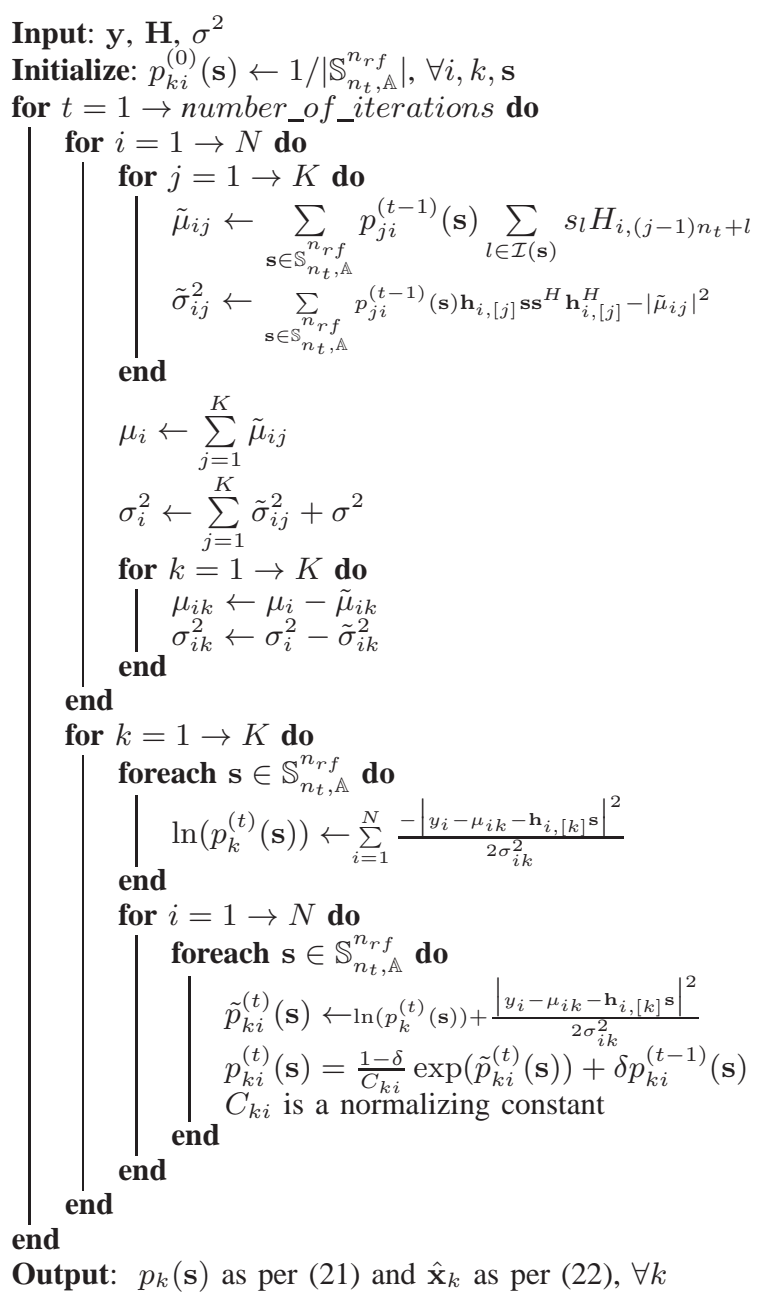

Algorithm 1: Listing of MP-GSM algorithm.

complexity of this MMSE detection for the system model in (4) is given by $O\left(N^{2} K n_{t}\right)$. In Table I, we present a complexity comparison between the MP-GSM and MMSE detection algorithms. From Table II it can be seen that the MP-GSM detection complexity is less than the MMSE detection complexity. In addition to having this complexity advantage over MMSE detection, MP-GSM detection achieves significantly better performance than MMSE detection (we will see this in the performance results presented next). We further note that the computation of double summation in (18) and 19 in the MP-GSM algorithm can further be simplified by using FFT, as the double summation can be viewed as a convolution operation.

Performance: We evaluated the BER performance of the MP-GSM detection algorithm in large-scale multiuser GSMMIMO systems by simulations. Here, we assume perfect channel state information (CSI) at the receiver. We will relax this assumption later. Figure 6 presents the performance of MP-GSM detection algorithm in a large-scale multiuser GSMMIMO system with the following system parameters: $K=16$, $N=64,128, n_{t}=4, n_{r f}=2$, and 4-QAM. Note that the spectral efficiency in this system is $6 \mathrm{bpcu}$ per user. We compare the performance of this system with two other systems which also have the same spectral efficiency of $6 \mathrm{bpcu}$ per user. These systems are: 1) conventional multiuser MIMO 
system with $n_{t}=n_{r f}=1$, 64-QAM, and ML detection using sphere decoding (note that this is massive MIMO system; we abbreviate it as M-MIMO in the figures), and 2) multiuser SM-MIMO system with $n_{t}=4, n_{r f}=1,16-\mathrm{QAM}$, and MP-GSM detection. From Fig. 6 we observe that GSMMIMO outperforms both SM-MIMO as well as conventional MIMO. For example, at a BER of $10^{-3}$, GSM-MIMO has a performance advantage of about $4 \mathrm{~dB}$ over SM-MIMO and about $7 \mathrm{~dB}$ over conventional MIMO. This observation is in conformance with similar performance advantage of GSMMIMO over SM-MIMO and conventional MIMO predicted by analytical upper bounds in Section $\amalg$

Performance comparison with other detectors: Next, in Fig. 7 we compare the performance of MP-GSM detection with that of MMSE detection in multiuser GSM-MIMO with $K=16, N=64,128, n_{t}=4, n_{r f}=2,4$-QAM, and 6 bpcu per user. From Fig. 7 we observe that the performance of MP-GSM algorithm is better than MMSE detection performance by $9 \mathrm{~dB}$ for $N=64$ and $3 \mathrm{~dB}$ for $N=128$ at a BER of $10^{-3}$. As noted in the discussion on complexity, MP-GSM achieves this better performance than MMSE at a lesser complexity than MMSE (as illustrated in Table III). Iterative detection/decoding schemes that use MMSE filters and provide further refinements are common in the literature [33]-[36]. In addition to the comparison with MMSE detection performance, Fig. 7 also presents a comparison with the performance of the MMSE-SIC detector and the multibranch MMSE decision feedback (MB-MMSE-DF) detector (with 4 branches and ordering) proposed in [36]. It can be seen that while the MB-MMSE-DF and MMSE-SIC detectors perform better than the MMSE detector, the proposed MPGSM detector outperforms the MMSE, MMSE-SIC, and MBMMSE-DF detectors. Moreover, the complexity of the MPGSM detector is less than those of the MMSE, MMSE-SIC, MB-MMSE-DF detectors.

\begin{tabular}{|c||c|c|c|}
\hline \multicolumn{1}{|c||}{} & \multicolumn{3}{c|}{$\begin{array}{c}\text { Complexity in number of real operations } \times 10^{6} \\
\left.\text { (GSM-MIMO with } N=128, n_{t}=4, n_{r f}=2,4-\mathrm{QAM}\right)\end{array}$} \\
\cline { 2 - 4 } & MMSE & $\begin{array}{c}\text { MP-GSM } \\
\text { (Sec. IV-A }\end{array}$ & $\begin{array}{c}\text { CHEMP-GSM } \\
\text { (Sec. IV-B }\end{array}$ \\
\hline \hline 16 & 3.594 & 2.195 & 3.142 \\
\hline 32 & 19.767 & 4.391 & 6.281 \\
\hline 64 & 28.355 & 8.782 & 12.265 \\
\hline 96 & 36.941 & 13.173 & 18.013 \\
\hline 128 & 45.526 & 17.564 & 21.637 \\
\hline
\end{tabular}

TABLE II

COMPARISON BETWEEN THE COMPLEXITIES (IN NUMBER OF REAL OPERATIONS) OF MMSE DETECTION, MP-GSM DETECTION, AND CHEMP-GSM DETECTION, IN MULTIUSER GSM MIMO WITH $N=128$, $n_{t}=4, n_{r f}=2$ AND 4-QAM.

Performance for same spectral efficiency and QAM size: We note that if both spectral efficiency and QAM size are to be kept same in GSM-MIMO and M-MIMO, then the number of spatial streams per user in M-MIMO has to increase. For example, GSM-MIMO can achieve 4 bpcu per user with 4QAM using $n_{t}=4$ and $n_{r f}=1$. M-MIMO can achieve the same spectral efficiency of 4 bpcu per user using one spatial stream (i.e., $n_{t}=1, n_{r f}=1$ ) with 16-QAM. But to achieve the same spectral efficiency using 4-QAM in MMIMO, we have to use $n_{t}=2, n_{r f}=2$, i.e., two spatial

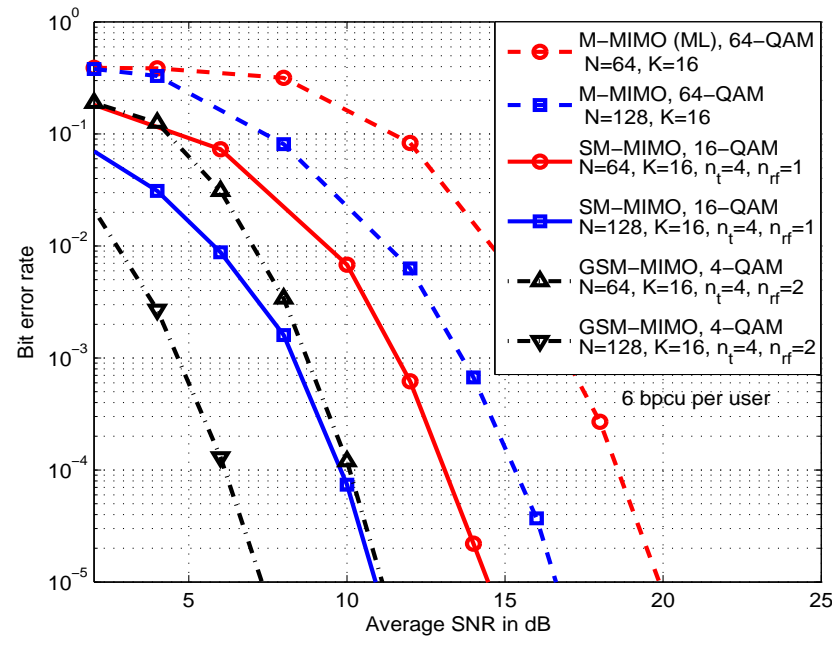

Fig. 6. BER performance of three different multiuser systems with the same spectral efficiency of 6 bpcu per user, $K=16, N=64,128: i$ ) M-MIMO with $n_{t}=n_{r f}=1$, 64-QAM, sphere decoding; $\left.i i\right)$ SM-MIMO with $n_{t}=4$, $n_{r f}=1$, 16-QAM, MP-GSM detection; iii) GSM-MIMO with $n_{t}=4$, $n_{r f}=2$, 4-QAM, MP-GSM detection.

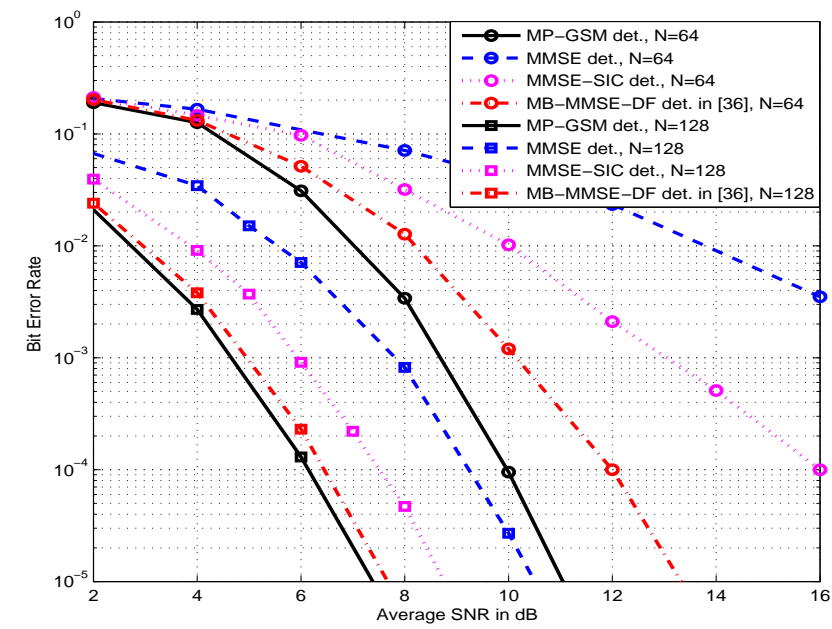

Fig. 7. BER performance comparison between $i$ ) MP-GSM detector, $i i)$ MMSE detector, iii) MMSE-SIC detector, and $i v$ ) MB-MMSE-DF detector in [36], in multiuser GSM-MIMO with $K=16, N=64,128, n_{t}=4$, $n_{r f}=2$, 4-QAM, and 6 bpcu per user.

streams per user with 4-QAM on each stream are needed. This increase in number of spatial streams per user increases the spatial interference. The effect of increase in number of spatial streams per user in M-MIMO for the same spectral efficiency on the performance is illustrated in Fig. 8 for $K=16$ and $N=128$.

In Fig. 8, we compare the performance of the following four systems with the same spectral efficiency of 4 bpcu per user, $K=16$, and $N=128: i)$ GSM-MIMO with $\left(n_{t}=4, n_{r f}=\right.$ 2, BPSK), ii) M-MIMO with ( $\left.\left.n_{t}=1, n_{r f}=1,16-\mathrm{QAM}\right), i i i\right)$ M-MIMO with ( $n_{t}=2, n_{r f}=2,4$-QAM), and $i v$ ) M-MIMO with ( $n_{t}=4, n_{r f}=4$, BPSK). Detection in the GSM-MIMO system is done using the MP-GSM algorithm. Detection in the M-MIMO systems is done using the likelihood ascent search (LAS) algorithm in [30] initialized with MMSE solution. It can be seen that among the four systems considered in Fig. 


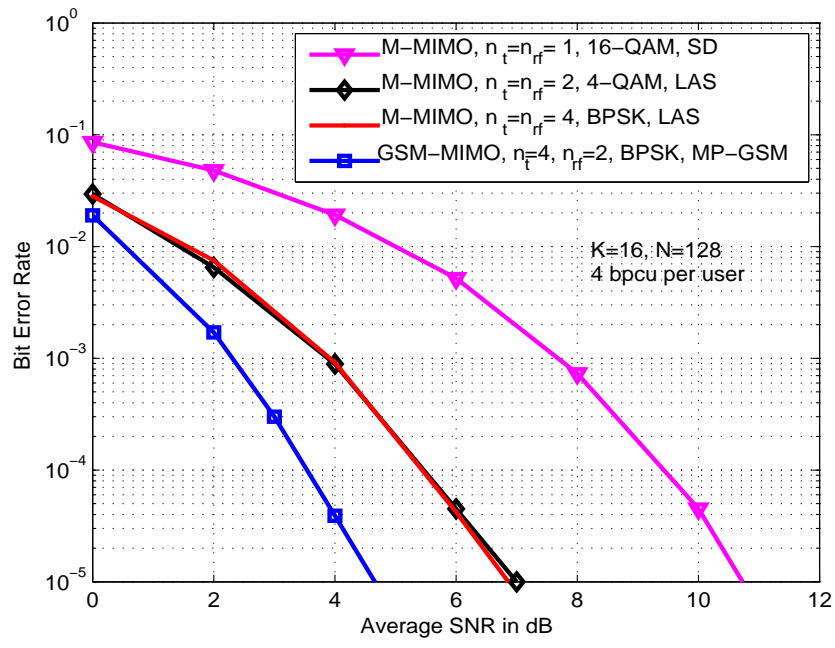

Fig. 8. BER performance of GSM-MIMO with $\left(n_{t}=4, n_{r f}=2\right.$, BPSK), M-MIMO with $\left(n_{t}=1, n_{r f}=1,16-\mathrm{QAM}\right)$, M-MIMO with $\left(n_{t}=2\right.$, $n_{r f}=2$, 4-QAM), and M-MIMO with $\left(n_{t}=4, n_{r f}=4\right.$, BPSK) for $K=16, N=128,4$ bpcu per user.

8 GSM-MIMO performs the best. This is because M-MIMO loses performance because of higher-order QAM or increased spatial interference from increased number of spatial streams per user.

In Fig. 9, we fix the number of users at $K=16$ and the spectral efficiency at 6 bpcu per user, vary the number of antennas $N$ at the $\mathrm{BS}$, and compare the SNRs required in various systems to achieve a target BER of $10^{-3}$. We compare the performance of the following four systems: $i$ ) GSM-MIMO with $\left(n_{t}=4, n_{r f}=2\right.$, 4-QAM), $\left.i i\right)$ SM-MIMO with $\left(n_{t}=4\right.$, $n_{r f}=1$, 16-QAM), $\left.i i i\right)$ SM-MIMO with $\left(n_{t}=2, n_{r f}=1\right.$, 32-QAM), and $i v)$ M-MIMO with $\left(n_{t}=1, n_{r f}=1\right.$, 64QAM). From Fig. 9, it can be observed that as the number of antennas at the BS increases, the required SNR to achieve the target BER decreases in all the four systems, which is expected because of the increased receive diversity. The sharp degradation observed for small values of $N$ is because the systems become under-determined when $K n_{t}>N$, and hence the required SNRs shoot up. When $K n_{t} \leq N$ (fully/overdetermined), GSM MIMO outperforms M-MIMO by about 9 $\mathrm{dB}$ and SM-MIMO by about 4 to $6 \mathrm{~dB}$.

\section{B. CHEMP-GSM detection algorithm}

In this subsection, we propose another detection algorithm based on message passing. We refer to the detection algorithm presented in this subsection as CHEMP-GSM (channel hardening exploiting message passing [24],[25] for GSM) algorithm. We also propose a novel channel estimator for use in the CHEMP-GSM detector. We refer to the CHEMP-GSM detector along with this channel estimator as the 'CHEMPGSM receiver'. CHEMP-GSM approach is another message passing approach which gives less complexity than MMSE but performs significantly better than MMSE.

Matched filtered system model: First, we perform a matched filter operation on the received signal vector $\mathbf{y}$ in (4) as $\mathbf{H}^{H} \mathbf{y}$,

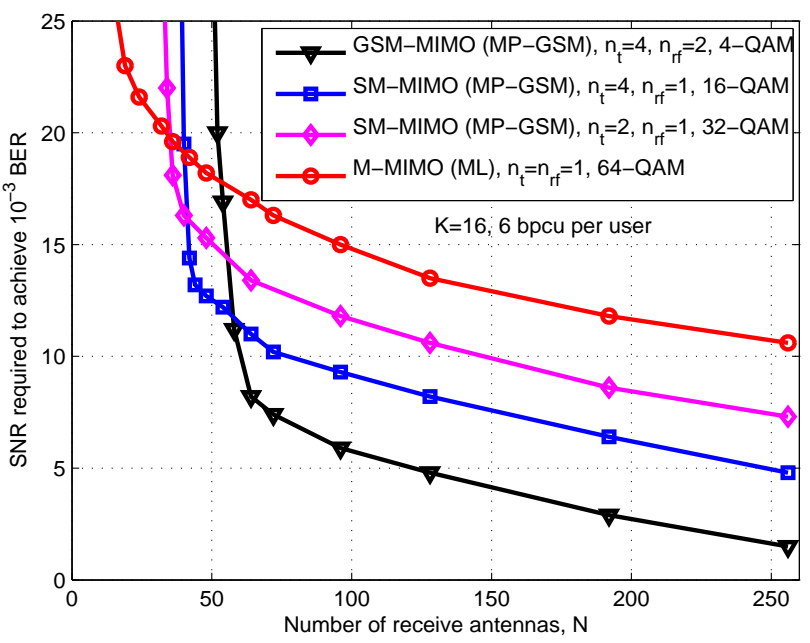

Fig. 9. Comparison of SNRs required by GSM-MIMO with $\left(n_{t}=4\right.$, $n_{r f}=2$, 4-QAM), SM-MIMO with $\left(n_{t}=4, n_{r f}=1\right.$, 16-QAM), SMMIMO with $\left(n_{t}=2, n_{r f}=1\right.$, 32-QAM), and M-MIMO with ( $n_{t}=1$, $n_{r f}=1$, 64-QAM) to achieve a target BER of $10^{-3}$ for $K=16$ and varying $N$, at the same spectral efficiency of $6 \mathrm{bpcu}$ per user.

which can be written as

$$
\mathbf{H}^{H} \mathbf{y}=\mathbf{H}^{H}(\mathbf{H} \mathbf{x}+\mathbf{n}) .
$$

A corresponding equivalent system model can be written as

where

$$
\mathbf{z}=\mathbf{J} \mathbf{x}+\mathbf{v},
$$

$$
\mathbf{z} \triangleq \frac{\mathbf{H}^{H} \mathbf{y}}{N}, \quad \mathbf{J} \triangleq \frac{\mathbf{H}^{H} \mathbf{H}}{N}, \quad \mathbf{v} \triangleq \frac{\mathbf{H}^{H} \mathbf{n}}{N} .
$$

Similar to $\mathbf{x}$ in the basic system model in (4), the vector $\mathbf{z}$ in (24) can be viewed as a concatenation of $K$ sub-vectors each

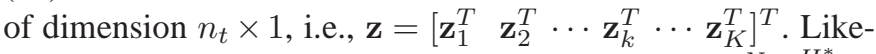
wise, $\mathbf{v}=\left[\begin{array}{llllll}\mathbf{v}_{1}^{T} & \mathbf{v}_{2}^{T} & \cdots & \mathbf{v}_{k}^{T} & \cdots & \mathbf{v}_{K}^{T}\end{array}\right]^{T}$, where $v_{j}=\sum_{i=1}^{N} \frac{H_{i j}^{*} n_{i}}{N}$ is the $j$ th element of $\mathbf{v}$ and $H_{i j}$ is the $(i, j)$ th element of $\mathbf{H}$. For large $N, v_{j}$ can be approximated to follow Gaussian distribution as $v_{j} \sim \mathcal{C N}\left(0, \sigma_{v}^{2}\right)$, where the variance $\sigma_{v}^{2}=\frac{\sigma^{2}}{N}$. Each sub-vector $\mathbf{z}_{k}$ can be expressed as

$$
\mathbf{z}_{k}=\mathbf{J}_{k k} \mathbf{x}_{k}+\underbrace{\sum_{j=1, j \neq k}^{K} \mathbf{J}_{k j} \mathbf{x}_{j}+\mathbf{v}_{k}}_{\triangleq \mathbf{g}_{k}},
$$

where $\mathbf{J}_{k j}$ is a $n_{t} \times n_{t}$ sub-matrix of $\mathbf{J}$ formed from the elements in rows $(k-1) n_{t}+1$ to $k n_{t}$ and columns $(j-1) n_{t}+1$ to $j n_{t}$, i.e., $\mathbf{J}$ can be written in terms of the sub-matrices as

$$
\mathbf{J}=\left[\begin{array}{cccc}
\mathbf{J}_{11} & \mathbf{J}_{12} & \cdots & \mathbf{J}_{1 K} \\
\mathbf{J}_{21} & \mathbf{J}_{22} & \cdots & \mathbf{J}_{2 K} \\
\vdots & & \ddots & \vdots \\
\mathbf{J}_{K 1} & \mathbf{J}_{K 2} & \cdots & \mathbf{J}_{K K}
\end{array}\right]
$$

The vector $\mathbf{g}_{k}$ defined in 26) denotes the interference-plusnoise to the $k$ th user's GSM signal. This term $\mathbf{g}_{k}$ involves the off-diagonal elements of $\mathbf{J}$ (i.e., $J_{k j}, k \neq j$ where $J_{k j}$ is the $(k, j)$ th element in $\mathbf{J})$. Due to channel hardening that occurs in large MIMO channels, the matrix $\mathbf{J}$ (and hence $\mathbf{J}_{k k}, \forall k$ ) has strong diagonal elements compared to off-diagonal terms for 


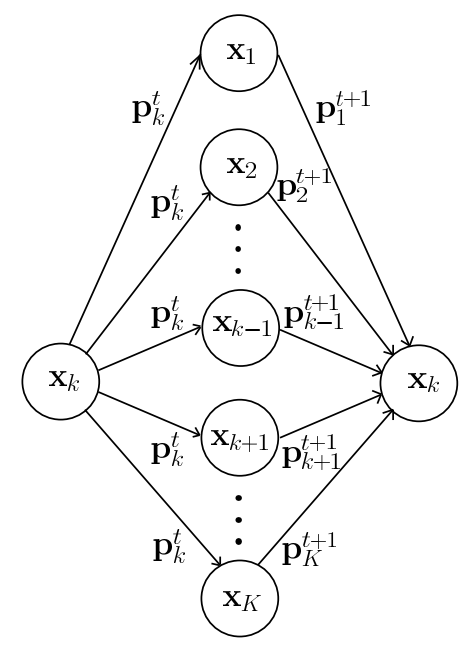

Fig. 10. Message passing in CHEMP-GSM algorithm.

large $N, K$. We approximate $\mathbf{g}_{k}$ to be multivariate Gaussian with mean $\boldsymbol{\mu}_{k}$ and covariance $\boldsymbol{\Sigma}_{k}$, which can be written as

$$
\begin{aligned}
& \boldsymbol{\mu}_{k}=\mathbb{E}\left(\mathbf{g}_{k}\right)=\sum_{j=1, j \neq k}^{K} \mathbf{J}_{k j} \mathbb{E}\left(\mathbf{x}_{j}\right) \\
& \boldsymbol{\Sigma}_{k}=\operatorname{Cov}\left(\mathbf{g}_{k}\right)=\sum_{j=1, j \neq k}^{K} \mathbf{J}_{k j} \operatorname{Cov}\left(\mathbf{x}_{j}\right) \mathbf{J}_{k j}^{H}+\sigma_{v}^{2} \mathbf{I}_{n_{t}},
\end{aligned}
$$

where

$$
\begin{aligned}
\mathbb{E}\left(\mathbf{x}_{j}\right) & =\sum_{\forall \mathbf{s}, \mathbf{s} \in \mathbb{S}_{n_{t}, \mathbb{A}}^{n_{r f}}} \mathbf{s} p_{j}(\mathbf{s}) \\
\operatorname{Cov}\left(\mathbf{x}_{j}\right) & =\sum_{\forall \mathbf{s}, \mathbf{s} \in \mathbb{S}_{n_{t}, \mathbb{A}}^{n_{r f}}} \mathbf{s s}^{H} p_{j}(\mathbf{s})-\mathbb{E}\left(\mathbf{x}_{j}\right) \mathbb{E}\left(\mathbf{x}_{j}\right)^{H}
\end{aligned}
$$

and

$$
p_{k}(\mathbf{s})=\operatorname{Pr}\left(\mathbf{x}_{k}=\mathbf{s}\right), \quad \mathbf{s} \in \mathbb{S}_{n_{t}, \mathbb{A}}^{n_{r f}} .
$$

Let $\mathbf{p}_{k}=\left[p_{k}\left(\mathbf{s}_{1}\right), p_{k}\left(\mathbf{s}_{2}\right), \cdots, p_{k}\left(\mathbf{s}_{\left|\mathbf{S}_{n_{+} n_{A} \mid}^{n_{n f}}\right|}\right)\right]$ denote the vector of probability masses corresponding to GSM signal vector $\mathbf{x}_{k}$. The probability masses $p_{k}(\mathbf{s})$ 's are approximated with the corresponding a posteriori probabilities (APP), i.e.,

$$
\begin{aligned}
p_{k}(\mathbf{s}) & \leftarrow \operatorname{Pr}\left(\mathbf{x}_{k}=\mathbf{s} \mid \mathbf{z}_{k}, \mathbf{J}\right) \\
& \propto \exp \left(\frac{-1}{2}\left(\mathbf{z}_{k}-\mathbf{J}_{k k} \mathbf{s}-\boldsymbol{\mu}_{k}\right)^{H} \mathbf{\Sigma}_{k}^{-1}\left(\mathbf{z}_{k}-\mathbf{J}_{k k} \mathbf{s}-\boldsymbol{\mu}_{k}\right)\right)
\end{aligned}
$$

Message passing: The system is modeled as a fullyconnected graph with $K$ nodes, where the $k$ th node is an approximate APP processor corresponding to $\mathbf{x}_{k}$. Node $k$ computes the APP based on the incoming messages and the knowledge of $\mathbf{z}_{k}$ and $\mathbf{J}$. The incoming messages to node $k$ are the APPs from the remaining nodes. The APP computed in node $k$, in turn, is passed to the remaining nodes for their APP computation in the next iteration. The messages exchanged between the nodes are illustrated in Fig. 10, The algorithm is as follows.

Step 1: The probability vectors $\mathrm{p}_{k}$ 's are initialized with equiprobable masses $1 /\left|\mathbb{S}_{n_{t}, \mathbb{A}}^{n_{r f}}\right|$.

Step 2: Node $k$ computes $\mathbf{p}_{k}$ as per (33) with the incoming vector messages $\left\{\mathbf{p}_{1}, \mathbf{p}_{2}, \cdots, \mathbf{p}_{k-1}, \mathbf{p}_{k+1}, \cdots, \mathbf{p}_{K}\right\}$. Damping of messages with damping factor $\delta \in[0,1)$ is done to

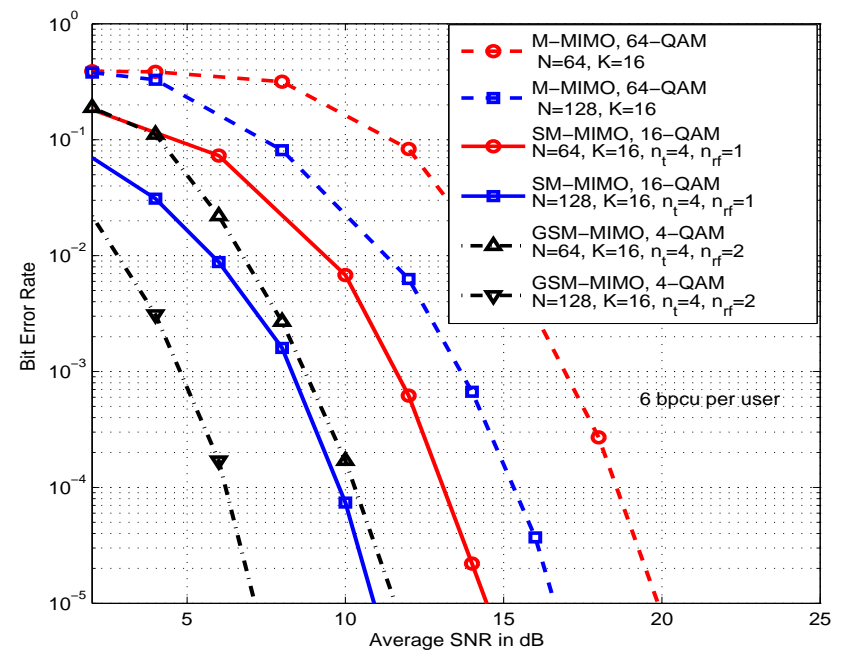

Fig. 11. BER performance of multiuser GSM-MIMO systems with $\left(n_{t}=4\right.$, $\left.n_{r f}=2,4-\mathrm{QAM}\right)$ and $\left(n_{t}=4, n_{r f}=1\right.$ using CHEMP-GSM detection, and M-MIMO system with $\left(n_{t}=1, n_{r f}=1,64-\mathrm{QAM}\right)$ using sphere decoding, at 4 bpcu per user, $K=16, N=64,128$.

improve the rate of convergence. That is, if $\tilde{\mathbf{p}}_{k}^{t}$ is the computed probability vector at the $t$ th iteration, the message at the end of $t$ th iteration is computed as

$$
\mathbf{p}_{k}^{t}=(1-\delta) \tilde{\mathbf{p}}_{k}^{t}+\delta \mathbf{p}_{k}^{t-1}
$$

Repeat Step 2 for a certain number of iterations, after which the algorithm stops. The estimate of the $l$ th modulation symbol transmitted by the $k$ th user is obtained as

$$
\hat{s}_{k, l}=\underset{s \in \mathbb{A}}{\operatorname{argmax}} \sum_{\forall \mathbf{s}, \mathbf{s} \in \mathbb{S}_{n_{t}, \mathbb{A}}^{n_{r f}}: \mathcal{X}_{l}(\mathbf{s})=s} p_{k}(\mathbf{s}),
$$

where $\mathcal{X}_{l}(\mathbf{s})$ is the $l$ th non-zero element in $\mathbf{s}$ and $l \in$ $\left\{1,2, \cdots, n_{r f}\right\}$. An estimate of the active antenna indices chosen for transmission by the $k$ th user is obtained as

$$
\hat{q}_{k}=\underset{q \in\{1, \cdots,|\mathcal{S}|\}}{\operatorname{argmax}} \sum_{\forall \mathbf{s}, \mathbf{s} \in \mathbb{S}_{n_{t}, \mathbb{A}}^{n_{r} f}: \mathcal{I}(\mathbf{s})=q} p_{k}(\mathbf{s}) .
$$

The values of $\hat{s}_{k, l}$ and $\hat{q}_{k}$ are then demapped to obtain the information bits of the $k$ th user.

Complexity: The orders of complexity for the computation of $\mathbf{z}$ and $\mathbf{J}$ are $O\left(N K n_{t}\right)$ and $O\left(N K^{2} n_{t}^{2}\right)$, respectively. The complexities for the computation of (27), (28) and (33) are of orders $O\left(n_{t}^{2} K^{2}\right), O\left(n_{t}^{3} K^{2}\right)$ and $O\left(n_{t}^{3} K\left|\mathbb{S}_{n_{t} \mathbb{A}}^{n_{r f}}\right|\right)$, respectively. For $N>K$ and $K>n_{t}$, the overall complexity of the algorithm is dominated by the computation of $\mathbf{J}$ whose complexity is $O\left(N K^{2} n_{t}^{2}\right)$. Therefore, the overall complexity of the algorithm is $O\left(N K^{2} n_{t}^{2}\right)$. This complexity is less than the MMSE detection complexity which is $O\left(N^{2} K n_{t}\right)$. This is illustrated numerically in Table $\amalg$ which shows the complexity comparison between MMSE and CHEMP-GSM detection.

Performance: In Fig. 11, we present the performance of the CHEMP-GSM detector at a spectral efficiency of 6 bpcu per user, $K=16, N=64,128$, assuming perfect CSI at the receiver. Figure 11 compares the performance of multiuser GSM-MIMO system with $\left(n_{t}=4, n_{r f}=2\right.$, 4-QAM), with 


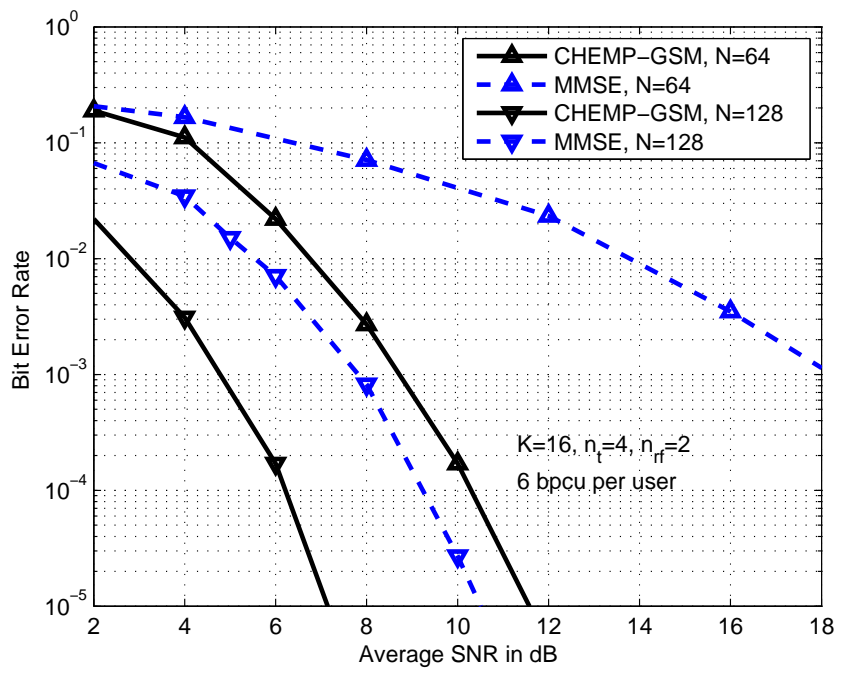

Fig. 12. BER performance comparison of multiuser GSM-MIMO using CHEMP-GSM detector and MMSE detector for $K=16, n_{t}=4, n_{r f}=2$, 4-QAM at 4 bpcu per user, $N=64,128$.

that of SM-MIMO system with $\left(n_{t}=4, n_{r f}=1,16\right.$ QAM) and M-MIMO system with $\left(n_{t}=1, n_{r f}=1\right.$, 64QAM). Detection in GSM-MIMO and SM-MIMO systems is done using CHEMP-GSM algorithm. Detection in M-MIMO system is done using sphere decoding. As observed in Sec. III-B and Sec. IV-A in Fig. 11 also we see that GSM-MIMO outperforms both SM-MIMO and M-MIMO. This is because of the smaller-sized QAM used in GSM-MIMO compared to those used in SM-MIMO and M-MIMO to achieve the same spectral efficiency.

CHEMP-GSM vs MMSE performance: Figure 12 shows the performance comparison between CHEMP-GSM detector and MMSE detector at a spectral efficiency of 6 bpcu per user, $K=16, n_{t}=4, n_{r f}=2$, and 4-QAM. It is observed that CHEMP-GSM detector outperforms MMSE detector by about $9 \mathrm{~dB}$ for $N=64$ and $3 \mathrm{~dB}$ for $N=128$ at a BER of $10^{-3}$. We note that CHEMP-GSM detector achieves this better performance at a lesser complexity compared to MMSE detector. This can be observed from Table 1 .

Performance as a function of loading factor: In Fig. 13, we compare the performance of different detectors for multiuser GSM-MIMO with $N=128, n_{t}=4, n_{r f}=2$, 4-QAM, and 6 bpcu per user as a function of system loading factor $K / N$. We plot the average SNR required by MP-GSM, CHEMP-GSM and MMSE detectors with perfect CSI to achieve a BER of $10^{-3}$. From Fig. 13, we observe that the proposed message passing based detectors outperform the MMSE detector by about 2 to $3 \mathrm{~dB}$ at lower loading factors. This gap widens as the system loading factor increases; for example, the gap is about $3 \mathrm{~dB}$ at $K / N=0.125$, and it widens to about $9 \mathrm{~dB}$ at $K / N=0.25$. The SNR required significantly increases for high loading factors because the channel gain matrix which is of dimension $N \times K n_{t}$ becomes under-determined for $K>\frac{N}{n_{t}}$. Also, both MP-GSM and CHEMP-GSM algorithms perform almost same, with MP-GSM having a lesser complexity than CHEMP-GSM (see Table (II). However, the matched filtered system model in CHEMP-GSM allows a

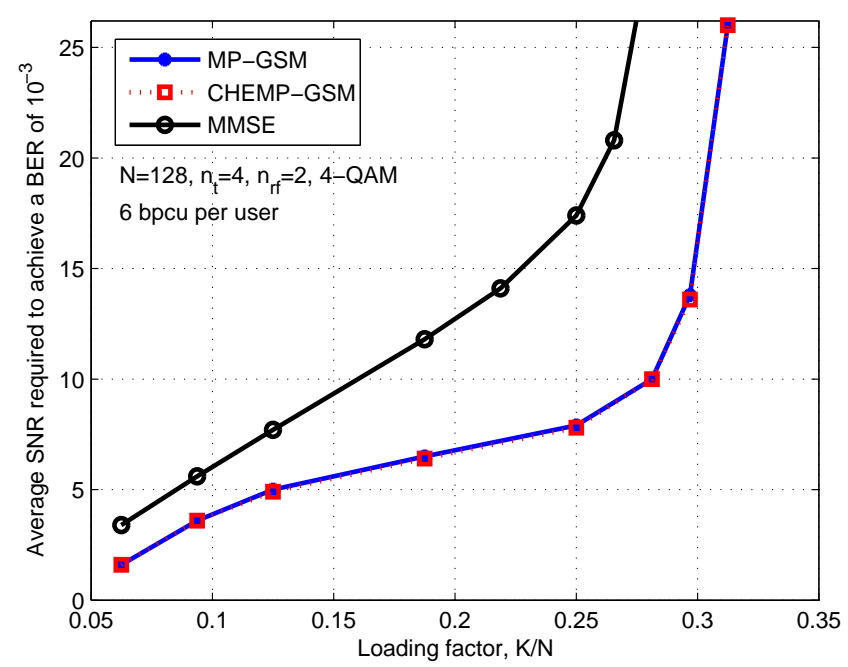

Fig. 13. SNR required by MP-GSM, CHEMP-GSM, and MMSE detectors to achieve a BER of $10^{-3}$ with $N=128, n_{t}=4, n_{r f}=2$, 4-QAM, and 6 bpcu per user, as a function of system loading factor $K / N$.

simple channel estimation technique, which is presented in the following subsection.

\section{Estimation of $\mathbf{H}^{H} \mathbf{H}$}

In obtaining the performance results reported in the previous subsection, we have assumed perfect CSI at the receiver. Now, we relax this assumption. We present a channel estimation scheme suited for use in CHEMP-GSM detector. A conventional approach is to directly obtain an estimated channel matrix $\hat{\mathbf{H}}$ through channel estimation techniques (MMSE channel estimation, for example) using pilot transmissions, and use $\widehat{\mathbf{H}}$ in place of $\mathbf{H}$ in detection algorithms. For the MMSE detector and MP-GSM detector, we follow this approach, and we call the receiver employing MMSE detector with MMSE channel estimate as 'MMSE receiver' and the receiver employing MP-GSM detector with MMSE channel estimate as 'MP-GSM receiver'. For CHEMP detector, however, instead of conventional approaches that estimate $\mathbf{H}$, we directly obtain an estimate of the matrix $\mathbf{J}$. The motivation for this approach is that $\mathbf{H}$ influences the proposed detection operation through $\mathbf{J}=\mathbf{H}^{H} \mathbf{H}$.

Assume that the channel is slowly fading, and that the fade remains constant for one frame duration. Each frame has $L_{f}$ channel uses, consisting of a pilot part and a data part. The pilot part consists of $K n_{t}$ channel uses, and the data part consists of $L_{f}-K n_{t}$ channel uses. Let $\mathbf{X}_{\mathrm{p}}=A \mathbf{I}_{K n_{t}}$ denote the pilot matrix, where, in the $j$ th channel use, $1 \leq j \leq$ $K n_{t}$, the $\left\lceil\frac{j}{n_{t}}\right\rceil$ th user terminal transmits a pilot symbol with amplitude $A$ through its antenna whose index is given by $((j-$ 1) $\left.\bmod n_{t}\right)+1$, and the other antennas remain silent. The signal received at the BS during pilot part is given by

$$
\mathbf{Y}_{\mathrm{p}}=\mathbf{H X}_{\mathrm{p}}+\mathbf{W}_{\mathrm{p}}=A \mathbf{H}+\mathbf{W}_{\mathrm{p}},
$$

where $A=\sqrt{K E_{s}}, E_{s}$ is the average symbol energy, and $\mathbf{W}_{\mathrm{p}}$ is the noise matrix. An estimate of the matrix $\mathbf{J}$ is obtained as

$$
\widehat{\mathbf{J}}=\frac{\mathbf{Y}_{\mathrm{p}}^{H} \mathbf{Y}_{\mathrm{p}}}{N A^{2}}-\frac{\sigma_{v}^{2}}{A^{2}} \mathbf{I}_{K n_{t}} \text {. }
$$




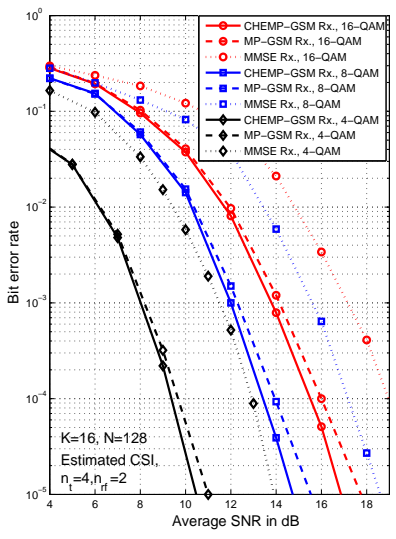

(a) BER vs SNR.

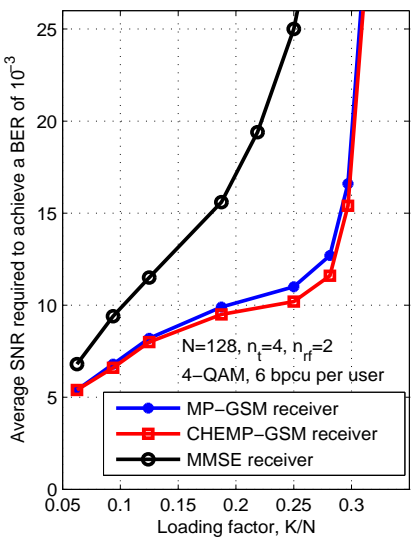

(b) SNR reqd. for $10^{-3} \mathrm{BER}$ vs $K / N$.
Fig. 14. Performance of MMSE Rx, MP-GSM Rx, and CHEMP-GSM Rx in multiuser GSM-MIMO with $N=128, n_{t}=4, n_{r f}=2$, 4-/8-/16-QAM, and estimated CSI.

where $\sigma_{v}^{2}=\frac{\sigma^{2}}{N}$. An estimate of the vector $\mathbf{z}$ is obtained as

$$
\widehat{\mathbf{z}}=\frac{\mathbf{Y}_{\mathrm{p}}^{H} \mathbf{y}}{N A},
$$

where $\mathbf{y}$ is the received signal vector in the data phase. These estimates $\widehat{\mathbf{J}}$ and $\widehat{\mathbf{z}}$ in 38 and (39) are used in the CHEMPGSM detection algorithm in place of $\mathbf{J}$ and $\mathbf{z}$.

Performance and complexity: In Figs. 14(a) and 14(b), we present performance comparisons between $i$ ) MMSE receiver (MMSE detector with MMSE channel estimate), $i i$ ) MP-GSM receiver (MP-GSM detector with MMSE channel estimate), and iii) CHEMP-GSM receiver (CHEMP-GSM detector with the proposed estimate of $\mathbf{J})$. Multiuser GSM-MIMO with $N=$ $128, n_{t}=4, n_{r f}=2$, and 4-/8-/16-QAM is considered. Figure 14(a) shows the BER vs SNR plots for $K=16$, i.e., loading factor $K / N=0.125$. Figure $14(\mathrm{~b})$ shows SNR required to achieve $10^{-3}$ BER as a function of loading factor $K / N$. Table III presents corresponding complexity comparison between the receivers considered. It can be observed that, because of the additional complexity of MMSE channel estimation, the complexities of MMSE receiver and MP-GSM receiver are more than the corresponding complexities of MMSE detector and MP-GSM detector in Table II However, the complexities of CHEMP-GSM receiver and CHEMP-GSM detector are the same. This is because the complexities of computing $\widehat{\mathbf{J}}$ and $\widehat{\mathbf{z}}$ are the same as those of computing $\mathbf{J}$ and $\mathbf{z}$, respectively. From Figs. 14(a) and 14(b) it can be seen that the performance of the CHEMP-GSM receiver is the best among the three receivers considered. Also, the complexities of both MP-GSM and CHEMP-GSM receivers are much less compared to that of MMSE receiver, and the complexity of CHEMP-GSM receiver is a little higher than the complexity of MP-GSM receiver due to the additional computation of $\widehat{\mathbf{z}}$ in 39 .

\section{Multiuser GSM-MiMO IN FREQUENCY SELECTIVE FADING}

In this section, we assume the multiuser GSM-MIMO system model described in Section II except for the channel

\begin{tabular}{|c||c|c|c|}
\hline \multicolumn{1}{|c||}{$K$} & Complexity in number of real operations $\times 10^{6}, N=128$ \\
\cline { 2 - 4 } & MMSE Rx. & MP-GSM Rx. & CHEMP-GSM Rx. \\
\hline \hline 16 & 4.041 & 2.466 & 3.142 \\
\hline 32 & 21.294 & 4.925 & 6.281 \\
\hline 64 & 30.037 & 10.831 & 12.265 \\
\hline 96 & 39.513 & 16.746 & 18.013 \\
\hline 128 & 48.622 & 20.961 & 21.637 \\
\hline \multicolumn{3}{|c|}{ TABLE III }
\end{tabular}

COMPARISON BETWEEN THE COMPLEXITIES (IN NUMBER OF REAL OPERATIONS ) OF THE MP-GSM RECEIVER, CHEMP-GSM RECEIVER, MMSE RECEIVER IN GSM-MIMO WITH $N=128, n_{t}=4, n_{r f}=2$, AND 4-QAM

model which is now assumed to be frequency selective. Let $L$ denote the number of multipath components between each pair of transmit antenna at the user and receive antenna at the BS. Let $H_{i,(k-1) n_{t}+j}^{(l)}$ denote the channel gain from the $j$ th transmit antenna of the $k$ th user to the $i$ th BS receive antenna on the $l$ th multipath component. The channel gains for the $l$ th multipath component are modeled as complex Gaussian with zero mean and variance $\Omega_{l}$. The power-delay profile of the channel is modeled as

$$
\begin{aligned}
\Omega_{l} & =\mathbb{E}\left[\left|H_{i,(k-1) n_{t}+j}^{(l)}\right|^{2}\right] \\
& =\Omega_{0} 10^{-\xi l / 10}, \quad l=0, \cdots, L-1 .
\end{aligned}
$$

where $\xi$ denotes the decay-rate of the average power in each of the multipath components in $\mathrm{dB}$. The total power of the channel is assumed to be unity, i.e., $\sum_{l=0}^{L-1} \Omega_{l}=1$. We will further assume that the channel coefficients are estimated at the BS using a pilot transmission based channel estimation.

CPSC transmission: We employ cyclic prefixed single carrier (CPSC) transmission, which has the advantage of low peak-to-average power ratio (PAPR) [31],[32]. Transmission is carried out in frames, where each frame consists of multiple blocks as shown in Fig. 15. The fade coefficients are assumed to be constant over one frame duration. Each frame consists of one pilot block (PB) meant for channel estimation, followed by $I$ data blocks (DB). The pilot block consists of $(L-1)+K n_{t} L$ channel uses. In the first $L-1$ channel uses in the pilot block, zeros are transmitted to avoid interference from previously transmitted frames. In each of the remaining $K n_{t} L$ channel uses, a $K n_{t}$-length pilot symbol vector comprising of pilot symbols transmitted from $K$ users ( $n_{t}$ pilot symbols per user) is received by the $N$ BS receive antennas. Each data block consists of $Q+L-1$ channel uses, where $Q$ information symbol vectors, each of length $K n_{t}$, prefixed by $(L-1)$ length cyclic prefix from each user are transmitted. With $I$ data blocks in a frame, the number of channel uses in the data part of the frame is $(Q+L-1) I$. Taking both pilot and data channel uses into account, the total number of channel uses per frame is $\left(K n_{t}+1\right) L+(Q+L-1) I-1$.

Channel estimation: Let $P$ be the power of the pilot symbol transmitted by each user. Then, in $K n_{t} L$ channel uses, the pilot sequence $\mathbf{p}_{j}^{k}$ transmitted by the $j$ th transmit antenna of the $k$ th user is given by the $K n_{t} L$-length vector $\mathbf{p}_{j}^{k}=\left[\begin{array}{lll}\mathbf{0}_{\left((k-1) n_{t}+j-1\right) L \times 1} & \sqrt{P} & \mathbf{0}_{\left(\left((K-k+1) n_{t}-j+1\right) L-1\right) \times 1}\end{array}\right]$. Let $\mathbf{h}_{i,(k-1) n_{t}+j}=\left[H_{i,(k-1) n_{t}+j}^{(0)} \cdots H_{i,(k-1) n_{t}+j}^{(l)} \cdots H_{i,(k-1) n_{t}+j}^{(L-1)}\right]$. 


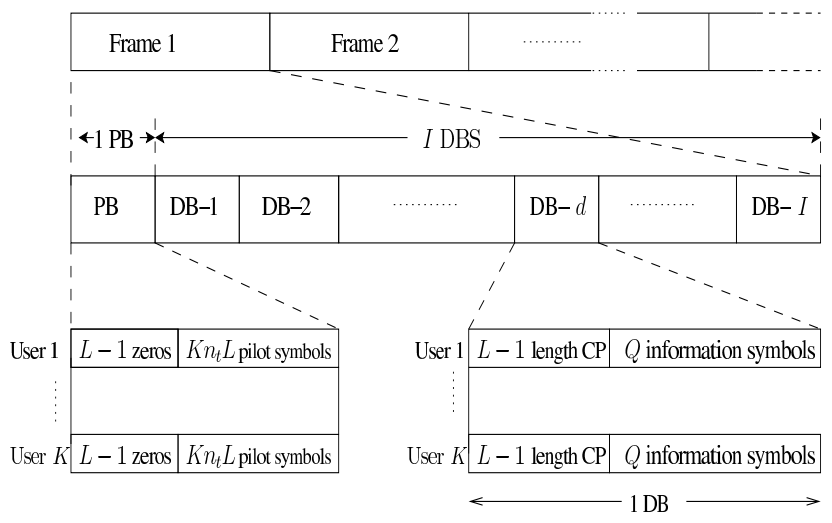

Fig. 15. Frame structure of CPSC scheme for multiuser GSM-MIMO in frequency selective fading.

The $K n_{t} L$-length vector received at the $i$ th BS antenna in the pilot phase is then given by

$$
\begin{aligned}
\mathbf{y}_{\mathrm{P}}^{i} & =\left[\begin{array}{llll}
y_{\mathrm{p}}^{i, 0} & y_{\mathrm{P}}^{i, 1} & \cdots & y_{\mathrm{P}}^{i, K n_{t} L-1}
\end{array}\right] \\
& =\underbrace{\left[\begin{array}{l}
\mathbf{h}_{i, 1}^{T} \cdots \mathbf{h}_{i,(k-1) n_{t}+j}^{T} \cdots \mathbf{h}_{i, K n_{t}}^{T}
\end{array}\right]^{T}}_{\triangleq \mathbf{h}^{i}} \sqrt{P}+\mathbf{n}_{\mathrm{p}}^{i},
\end{aligned}
$$

where $y_{\mathrm{P}}^{i, t}$ is the received signal at the $i$ th $\mathrm{BS}$ antenna in the $t$ th channel use of the pilot phase, and $\mathbf{n}_{\mathrm{p}}^{i}$ is the $K n_{t} L$-length noise sequence at the $i$ th BS antenna. An MMSE estimate of the channel gain vector $\mathbf{h}^{i}$ can be obtained from (41) as

$$
\widehat{\mathbf{h}}^{i}=\frac{\sqrt{P}}{P+\sigma^{2}} \mathbf{y}_{\mathrm{P}}^{i} .
$$

Signal detection: Each data block (DB) in a frame consists of a cyclic prefix (CP) followed by data symbols as shown in Fig. 15] Let $\mathbf{x}_{k}^{(t)} \in \mathbb{S}_{n_{t}, \mathbb{A}}^{n_{r f}}$ denote the transmit vector from the $k$ th user in the $t$ th channel use in a DB. The $k$ th user's transmit vector in a DB is of the form

$$
[\underbrace{\mathbf{x}_{k}^{(Q-L)^{T}} \mathbf{x}_{k}^{(Q-L+1)^{T}} \cdots \mathbf{x}_{k}^{(Q-1)^{T}}}_{\text {CP }} \underbrace{\mathbf{x}_{k}^{(0)^{T}} \mathbf{x}_{k}^{(1) T} \cdots \mathbf{x}_{k}^{(Q-1)^{T}}}_{\text {Data }}]^{T} .
$$

Assuming perfect synchronization and discarding the $\mathrm{CP}$ at the BS receiver, the received signal vector can be written as

$$
\mathbf{y}^{\prime}=\mathbf{H}^{\prime} \mathbf{x}^{\prime}+\mathbf{n}^{\prime}
$$

where $\mathbf{y}^{\prime}$ is $\left[\mathbf{y}^{(0)^{T}} \mathbf{y}^{(1)^{T}} \cdots \mathbf{y}^{(Q-1)^{T}}\right]^{T} \in \mathbb{C}^{N Q \times 1}, \mathbf{y}^{(t)} \in$ $\mathbb{C}^{N \times 1}$ denotes the received vector at the $t$ th channel use in a DB, $\mathbf{x}^{\prime}$ is $\left[\mathbf{x}^{(0)^{T}} \mathbf{x}^{(1)^{T}}, \cdots \mathbf{x}^{(Q-1)^{T}}\right]^{T} \in \mathbb{C}^{K n_{t} Q \times 1}, \mathbf{x}^{(t)} \in$ $\mathbb{C}^{K n_{t} \times 1}$ is the vector comprising of transmit vectors of all the users in the $t$ th channel use in a DB, $\mathbf{H}^{\prime}$ is the channel gain matrix of dimension $N Q \times K n_{t} Q$, and $\mathbf{n}^{\prime}$ is the additive white Gaussian noise vector given by $\left[\mathbf{n}^{(0)^{T}} \mathbf{n}^{(1)^{T}} \cdots \mathbf{n}^{(Q-1)^{T}}\right]^{T} \in$ $\mathbb{C}^{N Q \times 1}$. The received signal vector in the $t$ th channel use in a DB can be written as

$$
\mathbf{y}^{(t)}=\sum_{l=0}^{L-1} \mathbf{H}^{(l)} \mathbf{x}^{(t-l)}+\mathbf{n}^{(t)}, \quad t=0,1, \cdots, Q-1,
$$

where $\mathbf{H}^{(l)} \in \mathbb{C}^{N \times K n_{t}}$ is the channel gain matrix corresponding to the $l$ th multipath component such that $H_{i,(k-1) n_{t}+j}^{(l)}$ represents the channel gain from the $j$ th transmit antenna of the $k$ th user to the $i$ th BS receive antenna in the $l$ th multipath. For this system, the ML detection rule is given by

$$
\hat{\mathbf{x}}^{\prime}=\underset{\mathbf{x}^{\prime} \in \mathbb{G}^{Q}}{\operatorname{argmin}}\left\|\mathbf{y}^{\prime}-\mathbf{H}^{\prime} \mathbf{x}^{\prime}\right\|^{2},
$$

where $\mathbb{G} \triangleq\left(\mathbb{S}_{n_{t}, \mathbb{A}}^{n_{r f}}\right)^{K}$, and the exact computation of 445 requires exponential complexity in $K Q$. We shall formulate the system model in (43) into an equivalent system model in the frequency domain, and employ the algorithms for signal detection (presented in Section IV on the resulting equivalent system model.

It is noted that because of the addition of $\mathrm{CP}$, the matrix $\mathbf{H}^{\prime}$ is a block circulant matrix. Therefore, $\mathbf{H}^{\prime}$ can be transformed into a block diagonal matrix $\mathbf{D}$ as

$$
\mathbf{D}=\left(\mathbf{F} \otimes \mathbf{I}_{N}\right) \mathbf{H}^{\prime}\left(\mathbf{F}^{H} \otimes \mathbf{I}_{K n_{t}}\right),
$$

where $\mathbf{I}_{n}$ denotes $n \times n$ identity matrix, and $\mathbf{F}$ is the $Q \times Q$ DFT matrix, given by

$$
\mathbf{F}=\frac{1}{\sqrt{Q}}\left[\begin{array}{cccc}
\rho_{0,0} & \rho_{0,1} & \cdots & \rho_{0, Q-1} \\
\rho_{1,0} & \rho_{1,1} & \cdots & \rho_{1, Q-1} \\
\vdots & \vdots & \vdots & \vdots \\
\rho_{Q-1,0} & \rho_{Q-1,1} & \cdots & \rho_{Q-1, Q-1}
\end{array}\right],
$$

where $\rho_{u, v}=\exp \left(-\mathbf{j} \frac{2 \pi u v}{Q}\right)$. $\mathbf{D}$ is a block diagonal matrix of the form

$$
\mathbf{D}=\left[\begin{array}{ccc}
\mathbf{D}_{0} & \cdots & 0 \\
\vdots & \ddots & \vdots \\
0 & \cdots & \mathbf{D}_{Q-1}
\end{array}\right],
$$

where $\mathbf{D}_{q}$ is of dimension $N \times K n_{t}$. The $\left(i,(k-1) n_{t}+j\right)$ th element of $\mathbf{D}_{q}$ is the $q$ th element of the DFT of the vector $\left[H_{i,(k-1) n_{t}+j}^{(0)} H_{i,(k-1) n_{t}+j}^{(1)} \cdots H_{i,(k-1) n_{t}+j}^{(L-1)} 0 \cdots 0\right]^{T}$.

Performing DFT operation on the received vector $\mathbf{y}^{\prime}$ at the receiver, we get

$$
\mathbf{z}^{\prime}=\left(\mathbf{F} \otimes \mathbf{I}_{N}\right) \mathbf{y}^{\prime}=\left(\mathbf{F} \otimes \mathbf{I}_{N}\right) \mathbf{H}^{\prime} \mathbf{x}^{\prime}+\mathbf{w}^{\prime},
$$

where $\mathbf{w}^{\prime}=\left(\mathbf{F} \otimes \mathbf{I}_{N}\right) \mathbf{n}^{\prime}$. Further, $\mathbf{z}^{\prime}$ can be written as

$$
\begin{aligned}
\mathbf{z}^{\prime} & =\mathbf{D}\left(\mathbf{F} \otimes \mathbf{I}_{K n_{t}}\right) \mathbf{x}^{\prime}+\mathbf{w}^{\prime} \\
& =\overline{\mathbf{H}} \mathbf{x}^{\prime}+\mathbf{w}^{\prime},
\end{aligned}
$$

where $\overline{\mathbf{H}}=\mathbf{D}\left(\mathbf{F} \otimes \mathbf{I}_{K n_{t}}\right)$ is the equivalent channel. Now, detection can be performed on the system model in (49) using the MP-GSM and CHEMP-GSM algorithms presented in Section IV

Performance with perfect CSI: We evaluated the performance of multiuser GSM-MIMO CPSC systems in frequency selective channel with $L=3, Q=6, \xi=3 \mathrm{~dB}, N=128$, and perfect CSI. MMSE, MP-GSM and CHEMP-GSM algorithms are used for GSM-MIMO signal detection. Figure 16 shows the performance comparison between GSM-MIMO with $\left(n_{t}=4, n_{r f}=2,4-\mathrm{QAM}\right)$ and M-MIMO with $\left(n_{t}=1\right.$, $n_{r f}=1$, 64-QAM), both having 6 bpcu per user and $K=16$. LAS algorithm in [30] is used for M-MIMO signal detection. From Fig. 16, we observe that GSM-MIMO outperforms MMIMO by about $12 \mathrm{~dB}$ at a BER of $10^{-4}$. Also, MP-GSM and CHEMP-GSM detectors outperform MMSE detector by about $3 \mathrm{~dB}$ at a BER of $10^{-4}$. 


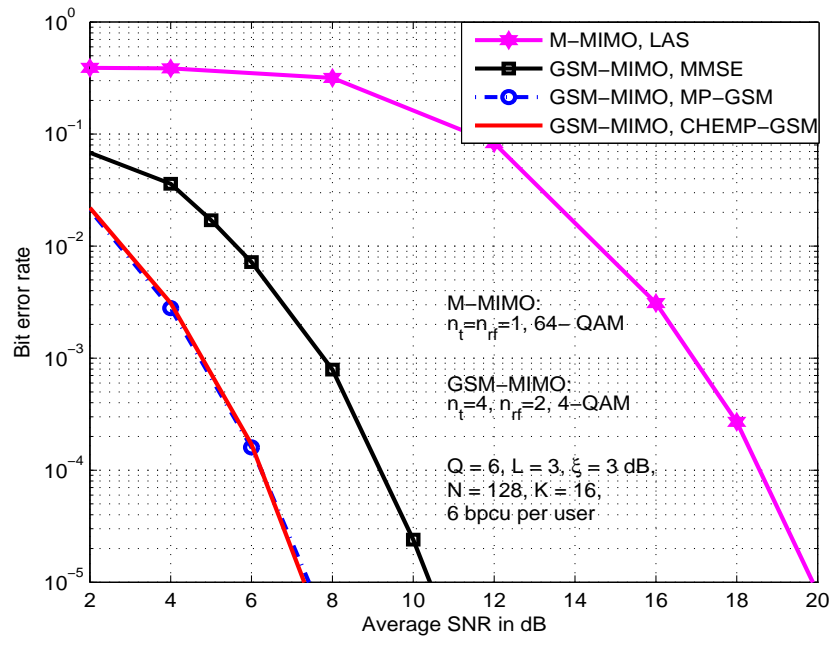

Fig. 16. BER performance of multiuser GSM-MIMO with $\left(n_{t}=4, n_{r f}=\right.$ 2 , 4-QAM) and M-MIMO with ( $\left.n_{t}=1, n_{r f}=1,64-\mathrm{QAM}\right)$ for $K=16$, $N=128,6$ bpcu per user, frequency selective fading with $L=3$ and $\xi=3$ $\mathrm{dB}$, CPSC transmission with $Q=6$, and perfect CSI

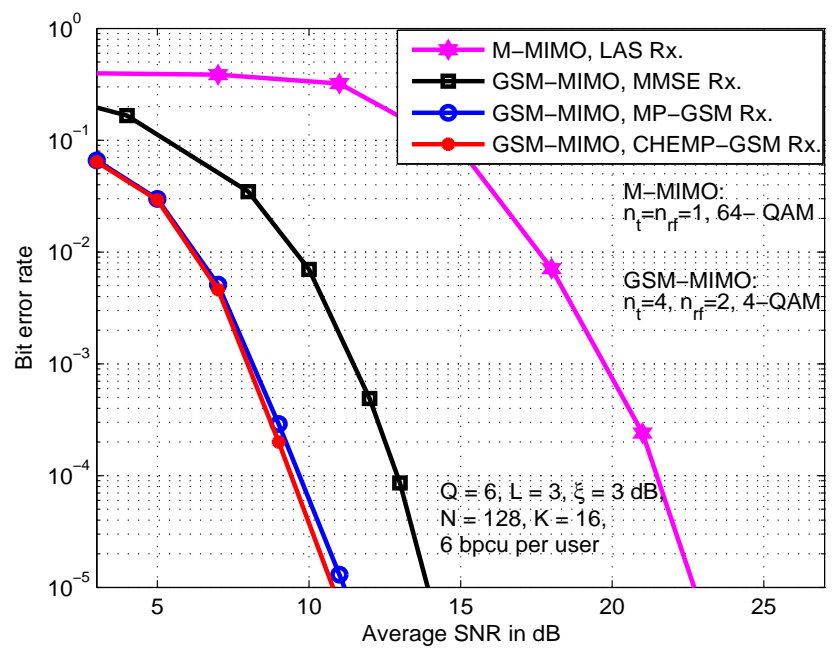

Fig. 17. BER performance of multiuser GSM-MIMO with $\left(n_{t}=4, n_{r f}=\right.$ 2 , 4-QAM) and M-MIMO with $\left(n_{t}=1, n_{r f}=1,64-\mathrm{QAM}\right)$ for $K=16$, $N=128,6$ bpcu per user, frequency selective fading with $L=3, \xi=3$ $\mathrm{dB})$, CPSC transmission with $(Q=6, L=3, \xi=3 \mathrm{~dB})$, estimated CSI.

Performance with estimated CSI: Figure [17 shows the performance of GSM-MIMO CPSC systems with estimated CSI for the same system and channel parameters in Fig. 16 MMSE receiver (MMSE detector with MMSE channel estimator), MP-GSM receiver (MP-GSM detector with MMSE channel estimate, and CHEMP-GSM receiver (CHEMP-GSM detector with the proposed estimate of $\mathbf{J}$ ) are used for GSMMIMO. LAS receiver (LAS detection with MMSE channel estimator) is used for M-MIMO. We observe that GSM-MIMO CPSC system performs better than M-MIMO CPSC system by about $11 \mathrm{~dB}$ at a BER of $10^{-4}$.

\section{Vi. CONCLUSions}

We investigated generalized spatial modulation (GSM) for multiuser communication on the uplink in large-scale MIMO systems. We derived an analytical upper bound on the average bit error probability in multiuser GSM-MIMO systems with
ML detection. The bound was shown to be tight at moderateto-high SNRs. Numerical results showed that, for the same spectral efficiency, multiuser GSM-MIMO can outperform conventional multiuser MIMO by several dBs. We also proposed low-complexity algorithms for multiuser GSM-MIMO signal detection and channel estimation at the BS receiver based on message passing. The performance of these proposed algorithms in large-scale GSM-MIMO systems with tens of users and hundreds of BS antennas showed that multiuser GSM-MIMO can outperform conventional multiuser MIMO. The SNR advantage of GSM-MIMO over conventional MIMO is attributed to the following reasons: $(i)$ because of the spatial index bits, to achieve the same spectral efficiency, GSMMIMO can use a lower-order QAM alphabet compared to that in conventional MIMO, and (ii) to achieve same spectral efficiency and QAM size, conventional MIMO will need more spatial streams per user which results in increased spatial interference. This performance advantage along with low RF hardware complexity makes large-scale multiuser GSMMIMO very attractive. We further note that the SM concept has recently been validated with the aid of experimental activities in indoors and outdoors [37],[38]. These practical advancements in SM and the performance advantage in GSMMIMO suggest that large-scale multiuser GSM-MIMO is an attractive technology for future wireless systems like 5G.

\section{REFERENCES}

[1] A. Chockalingam and B. S. Rajan, Large MIMO Systems, Cambridge Univ. Press, Feb. 2014.

[2] F. Rusek, D. Persson, B. K. Lau, E. G. Larsson, T. L.Marzetta, O. Edfors, and F. Tufvesson, "Scaling up MIMO: opportunities and challenges with very large arrays," IEEE Signal Process. Mag., vol. 30, no. 1, pp. 40-60, Jan. 2013.

[3] R. C. de Lamare, "Massive MIMO systems: signal processing challenges and research trends," available online: arXiv:1310.7282 [cs.IT] 28 Oct 2013.

[4] E. G. Larsson, O. Edfors, F. Tufvesson, and T. L. Marzetta, "Massive MIMO for next generation wireless systems," IEEE Commun. Mag., vol. 52, no. 2, pp. 186-195, Feb. 2014.

[5] M. Di Renzo, H. Haas, and P. M. Grant, "Spatial modulation for multiple-antenna wireless systems: a survey," IEEE Commun. Mag., vol. 50, no. 12, pp. 182-191, Dec. 2011.

[6] M. Di Renzo, H. Haas, A. Ghrayeb, S. Sugiura, and L. Hanzo, "Spatial modulation for generalized MIMO: challenges, opportunities and implementation," Proceedings of the IEEE, vol. 102, no. 1, pp. 56103, Jan. 2014

[7] P. Yang, M. Di Renzo, Y. Xiao, S. Li, and L. Hanzo, "Design guidelines for spatial modulation," IEEE Commun. Surveys \& Tutorials. doi:10.1109/COMST.2014.2327066.

[8] M. Di Renzo and H. Haas, "A general framework for performance analysis of space shift keying (SSK) modulation in MISO correlated Nakagami- $m$ fading channels," IEEE Trans. Commun., vol. 58, no. 9, pp. 2590-2603, Sep. 2010.

[9] M. Di Renzo and H. Haas, "Bit error probability of SM-MIMO over generalized fading channels," IEEE Trans. Veh. Tech., vol. 61, no. 3, pp. 1124-1144, Mar. 2012.

[10] M. Di Renzo and H. Haas, "On transmit diversity for spatial modulation MIMO: impact of spatial constellation diagram and shaping filters at the transmitter," IEEE Trans. Veh. Tech., vol. 62, no. 6, pp. 2507-2531, Jul. 2013.

[11] S. Narayanan, M. J. Chaudhry, A. Stavridis, M. Di Renzo, F. Graziosi, and H. Haas, "Multi-user spatial modulation MIMO," in Proc. IEEE WCNC 2014, pp. 671-676, Apr. 2014.

[12] M. Di Renzo and H. Haas, "Bit error probability of space-shift keying MIMO over multiple-access independent fading channels," IEEE Trans. Veh. Tech., vol. 60, no. 8, pp. 3694-3711, Oct. 2011. 
[13] N. Serafimovski, S. Sinanovic, A. Younis, M. Di Renzo, and H. Haas, "2-user multiple access spatial modulation," in Proc. IEEE GLOBECOM'2011 Workshop - HeterWMN 2011, Dec. 2011.

[14] N. Serafimovski1, S. Sinanovic, M. Di Renzo, and H. Haas, "Multiple access spatial modulation," EURASIP J. Wireless Commun. and Networking 2012. doi:10.1186/1687-1499-2012-299.

[15] T. L. Narasimhan, P. Raviteja, and A. Chockalingam, "Large-scale multiuser SM-MIMO versus massive MIMO," in Proc. ITA 2014, Feb. 2014.

[16] P. Raviteja, T. L. Narasimhan, and A. Chockalingam, "Detection in largescale multiuser SM-MIMO systems: algorithms and performance," in Proc. IEEE VTC 2014-Spring, May 2014.

[17] J. Wang, S. Jia, and J. Song, "Generalised spatial modulation system with multiple active transmit antennas and low complexity detection scheme," IEEE Trans. Wireless Commun., vol. 11, no. 4, pp. 1605-1615, Apr. 2012.

[18] T. Datta and A. Chockalingam, "On generalized spatial modulation," in Proc. IEEE WCNC 2013, Apr. 2013.

[19] P. Wang and T. Le-Ngoc, "A low-complexity generalized sphere decoding approach for underdetermined linear communication systems: performance and complexity evaluation," IEEE Trans. Commun., vol. 57, no. 11, pp. 3376-3388, Nov. 2009.

[20] A. Younis, S. Sinanovic, M. Di Renzo, R. Mesleh, and H. Haas, "Generalized sphere decoding for spatial modulation," IEEE Trans. Commun., vol. 61, no. 7, pp. 2805-2815, Jul. 2013.

[21] M. S. Alouini and A. Goldsmith, "A unified approach for calculating error rates of linearly modulated signals over generalized fading channels," IEEE Trans. Commun., vol. 47, no. 9, pp. 1324-1334, Sep. 1999.

[22] D. Tse and P. Viswanath, Fundamentals of Wireless Communication, Cambridge Univ. Press, 2005.

[23] A. Tulino and S. Verdu, "Random matrix theory and wireless communications," Foundations and Trends in Commun. and Inf. Theory, vol. 1, no. 1, pp. 1-182, Jun. 2004. doi: 10.1561/0100000001.

[24] T. L. Narasimhan and A. Chockalingam, "Channel hardening-exploiting message passing (CHEMP) receiver in large-scale MIMO systems," IEEE J. Sel. Topics in Signal Process., vol. 8, no. 5, pp. 847-860, Oct. 2014.

[25] T. L. Narasimhan and A. Chockalingam, "CHEMP receiver for largescale multiuser MIMO systems using spatial modulation," in Proc. EUSIPCO'2014, Sep. 2014.

[26] M. Pretti, "A message passing algorithm with damping," $J$ Stat. Mech.: Theory and Practice, Nov. 2005. doi:10.1088/17425468/2005/11/P11008

[27] H. Wymeersch, F. Penna, and V. Savic, "Uniformly reweighted belief propagation for estimation and detection in wireless networks," IEEE Trans. Wireless Commun., vol. 11. no. 4, pp. 1587-1595, Apr. 2012.

[28] J. Liu and R. C. de Lamare, "Low-latency reweighted belief propagation decoding for LDPC codes," IEEE Commun. Lett., vol. 16, no. 10, pp. 1660-1663, Oct. 2012.

[29] Y. Gong, X. Liu, W. Yecai, and G. Han, "Effective informed dynamic scheduling for belief propagation decoding of LDPC codes," IEEE Trans. Commun., vol. 59, no. 10, pp. 2683-2691, Oct. 2011.

[30] K. V. Vardhan, S. K. Mohammed, A. Chockalingam, and B. S. Rajan, "A low-complexity detector for large MIMO systems and multicarrier CDMA systems," IEEE J. Sel. Areas in Commun., vol. 26, no. 3, pp. 473-485, Apr. 2008.

[31] B. Muquet, Z. Wang, G. B. Giannakis, M. de Courville, and P. Duhamel, "Cyclic prefixing or zero padding for wireless multicarrier transmissions?," IEEE Trans. Commun., vol. 50, no. 12, pp. 2136-2148, Dec. 2002.

[32] S. Ohno, "Performance of single-carrier block transmissions over multipath fading channels with linear equalization," IEEE Trans. Signal Process., vol. 54, no. 10, pp. 3678-3687, Oct. 2006.

[33] J. Karjalainen, N. Veselinovic, K. Kansanen, and T. Matsumoto, "Iterative frequency domain joint-over-antenna detection in multiuser MIMO," IEEE Trans. Commun., vol. 6, no. 10, pp. 3620-3631, Oct. 2007.

[34] S. Yang, T. Lv, R. Maunder, and L. Hanzo, "Unified bit-based probabilistic data association aided MIMO detection for high-order QAM constellations," IEEE Trans. Veh. Tech., vol. 60, no. 3, pp. 981-991, Mar. 2011.

[35] J. W. Choi, A. C. Singer, J Lee, and N. I. Cho, "Improved linear softinput soft output detection via soft feedback successive interference cancellation," IEEE Trans. Commun., vol. 58, no. 3, pp. 986-996, Mar. 2010.

[36] R. C. de Lamare, "Adaptive and iterative multi-branch MMSE decision feedback detection algorithms for multi-antenna Systems," IEEE Trans. Wireless Commun., vol. 12, no. 10, pp. 5294-5308, Oct. 2013.
[37] N. Serafimovski, A. Younis, R. Mesleh, P. Chambers, M. Di Renzo, C.-X. Wang, P. M. Grant, M. A. Beach, and H. Haas, "Practical implementation of spatial modulation," IEEE Trans. Veh. Tech., vol. 62, no. 9 , pp. 4511-4523, Nov. 2013.

[38] A. Younis, W. Thompson, M. Di Renzo, C.-X. Wang, M. A, Beach, H. Haas, and P. M. Grant, " Performance of spatial modulation using measured real-world channels," in Proc. IEEE VTC 2013-Fall, Sep. 2013. 